\author{
Modelling drainage \\ of the precorneal tear film \\ after a blink
}

\section{R.J. Braun*}

Faculty of Mathematical Studies

University of Southampton

Southampton SO17 1BJ, UK

A.D. Fitt

Faculty of Mathematical Studies

University of Southampton

Southampton SO17 1BJ, UK

Technical Report No. 2002-16

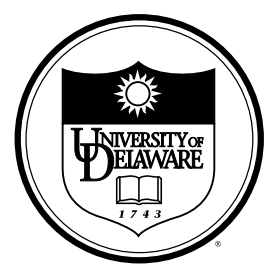

DEPARTMENT

$\mathrm{OF}$

MATHEMATICAL SCIENCES

University of Delaware

Newark, Delaware

*Permanent Address: Department of Mathematical Sciences, University of Delaware, Newark, DE 19716, USA; braun@math.udel.edu 


\title{
Modelling drainage of the precorneal tear film after a blink
}

\author{
R.J. BRAUN ${ }^{* 1}$ AND A.D. FitT ${ }^{1}$ \\ ${ }^{1}$ Faculty of Mathematical Studies, University of Southampton, Southampton \\ SO17 1BJ, UK
}

\begin{abstract}
We study the drainage of the precorneal tear film in humans. A fluid dynamic model for the drainage of the aqueous layer is developed that includes the effects of evaporation and gravity. The model may be reduced to a single nonlinear partial differential equation for the thickness of the aqueous layer. The equation is solved numerically and accurate times for film rupture are obtained for physically realistic parameters. The results indicate that though gravity and evaporation are not the most dominant effects in some parts of the film, they can nevertheless materially affect the film drainage process and should therefore be included in models for tear film drainage.
\end{abstract}

\section{Introduction}

In this study, we wish to investigate the drainage of the human tear film once the eyelid has opened after a blink. The tear film is essential to provide a perfect optical surface for the eye. It also acts as a lubricating layer during blinking and contains enzymes such as lysozyme and beta lysin that have bacteriocidal properties (Holly \& Lemp [16]). A typical interblink time for healthy humans is 5-8s (Berger \& Corrsin [2]), but interblink times may vary widely for different animals (for example, a typical interblink time for a rabbit is 10 minutes). Improved quantitative models for both blinking and tear film drainage are essential for understanding the behaviour of both normal eyes and eyes affected by the multitude of conditions known as dry eye (Bron [7]; Lemp [18]; Rolando \& Zierhut [30]). The causes of dry eye are many and various, but previous studies (see, for example, Bron [7], Lemp [18], Rolando \& Zierhut [30] and many others)

*Permanent Address: Department of Mathematical Sciences, University of Delaware, Newark, DE 19716, USA; braun@math.udel.edu 
have identified two main classes of the condition, namely insufficient tear film production, and increased tear film evaporation compared to normal eyes.

The human tear film is most often described as consisting of three distinct layers (Ehlers [12]; Mishima [22]; Wilson [38]). A mucus layer lies on top of the epithelial cells of the cornea; an aqueous layer is present above the mucus layer, and a lipid or fatty layer covers the aqueous layer.

The mucus layer that overlays the corneal epithelial cells has a typical thickness of $0.5-1 \mu \mathrm{m}$ (Rolando \& Refojo [29], Wong et al. [40]). A long-held view was that the corneal surface itself was not wettable by water and that the mucus layer was necessary for the wetting of the cornea (see, for example, Holly $[15])$. However, this view appears now to have been largely discredited and it is generally accepted that both the cornea and the mucus are hydrophilic (Sharma [32], Sharma [33]). Notwithstanding this, the mucus may still help to promote wetting of the cornea by more subtle means (for example, by transporting away nonwettable debris). The mucus is 10 to 100 times more viscous than the overlying aqueous layer (Sharma et al. [34]), but it may nevertheless slip on the surface of the cornea (Sharma [33]). There is some evidence that the mucus layer may itself rupture, and this may have implications for tear film stability in humans; such rupture was observed in an analogue system (water over silicone oil) investigated by Sharma et al. [34].

The aqueous layer is 4 to $10 \mu \mathrm{m}$ thick (Mishima [22]; Sharma [33] and references therein). It is supplied by the main and accessory lacrimal glands. It is primarily composed of water, with up to about $2 \%$ impurities (Fatt \& Weissman [13]) including inorganic salts, glucose, urea, proteins and glycoproteins (Holly \& Lemp [16]). It is often assumed to have the properties of pure water, though it has been proposed that it may be both viscoelastic and shear thinning (Bron \& Tiffany [8]; Tiffany [37]). The case for considering the aqueous and mucus layers as a single layer was made by Rolando \& Zierhut [30], for example; however, in Holly [15] and Nagyová \& Tiffany [24], virtually no dissolved mucus was found in tears extracted from humans. In section 2 we will therefore formulate a model for the drainage of an aqueous layer that emerges from a three-layer view of the tear film.

The lipid or fatty layer that lies above the aqueous layer is about $0.1-$ $-0.2 \mu \mathrm{m}$ thick (Berger \& Corrsin [2]; Franck [14]; Norn [26]). For patients with conjunctivitis or contact lens wearers it may be significantly thicker. The lipid layer serves two main functions; it greatly reduces evaporation and lowers the surface tension of the tear film, thus increasing its stability. The lipid layer is expressed from meibomian glands that are located on the upper eyelids through slits on the lid margins, and possibly also by the Zeis and Moll glands (Holly \& Lemp [16]). It has been suggested (McCulley \& Shine [20]) that the lipid layer is composed of two regions, namely a thin polar, surface-active spatially-ordered component located at the interface between the aqueous and the lipid layers, and a thicker overlying apolar component.

As noted above, the lipid layer plays a critical role in tear film stability by 
reducing evaporation (Mishima \& Maurice [23]). A number of previous studies have performed in vivo measurements of tear film evaporation. The average rate of evaporation in normal eyes was found to be about $4 \times 10^{-6} \mathrm{~kg} / \mathrm{m}^{2} / \mathrm{s}$, but increased to $8 \times 10^{-6} \mathrm{~kg} / \mathrm{m}^{2} / \mathrm{s}$ for dry eyes (Rolando \& Refojo [29]). More recent measurements by Mathers [19] reported average evaporation rates of $15 \times$ $10^{-6} \mathrm{~kg} / \mathrm{m}^{2} / \mathrm{s}$ for normal eyes and up to $60 \times 10^{-6} \mathrm{~kg} / \mathrm{m}^{2} / \mathrm{s}$ for dry eyes, and led to a hypothesis that for patients with meibomian gland loss evaporation may be a major cause of dry eye. Despite the fact that evaporation is a main classification criterion of the causes of dry eye (Lemp [18]), it has been argued on the basis of the data of Rolando \& Refojo [29] that the contribution of evaporation is negligible. Creech et al. [10] used a diffusion-limited model for evaporative transport to make this case. However, Holly \& Lemp [16] estimate that though about $90 \%$ of the lacrimal fluid is removed by the excretory system, the other $10 \%$ evaporates between blinks. Together with the newer data published by Mathers [19], it appears to us that evaporation may yet have a role to play in tear film stability; this will be investigated further below.

The lipid layer also reduces the surface tension of the tear film. In an effort understand the role of lipid layer components various analogues for human tear film components were studied by Holly [15] in order to develop a theory for the formation of tear films. More recently, actual human tear film components have been evaluated for their effect on the surface tension of tears and have been compared with animal analogues (Nagyová \& Tiffany [24]). These more recent measurements reported a value of $43.3 \mathrm{mN} / \mathrm{m}$ for the surface tension between the tear film and air, while prior measurements have given values of $46 \mathrm{mN} / \mathrm{m}$ (Miller [21]); this is a significant reduction from the value of $72.3 \mathrm{mN} / \mathrm{m}$ for a standard pure water/air interface. McCulley \& Shine [20] reasoned that the polar layer of the lipid region is mostly responsible for this effect.

An important quantitative clinical tool used by ophthalmologists to quantify dry eye conditions is the tear film break-up time (BUT). (Exact definitions of BUT vary from study to study, but all essentially measure the time taken for a dry patch to appear on the surface of the cornea. This matter will be discussed further below.) BUTs are reduced for all forms of dry eye compared to normal eyes (Bron [7]; Holly \& Lemp [16]). A key aim of this study is to show that addition of gravity and evaporation effects to those of capillarity predicts very realistic BUTs from a relatively simple hydrodynamic theory derived from wellknown equations of fluid dynamics.

In vivo measurements of geometrical tear film quantities have been made for the tear meniscus height (TMH) and the tear meniscus curvature (TMC). TMH measurements were performed by Port \& Asaria [28] in order to assess total tear film volume; Doughty et al. [11] measured TMH in elderly Caucasians. Reported TMH values range from $0.18 \mathrm{~mm}$ to $0.41 \mathrm{~mm}$ (Port \& Asaria [28]); an average value of about $0.33 \mathrm{~mm}$ seems to be reasonable.

TMC measurements have been made using reflective meniscometry by Yokoi et al. [41] in order to correlate TMC with dry eye conditions. Creech et al. [10] 
used a profile view and digital processing to measure TMC, and found an average radius of curvature of about $0.35 \mathrm{~mm}$. They then computed tear film thicknesses away from the meniscus using the results of Wong et al. [40], obtaining results that ranged from $2.8 \mu \mathrm{m}$ to $24 \mu \mathrm{m}$.

Previous theoretical efforts to describe the mathematics of tear film formation have, in our opinion, been mainly successful, though much still remains to be accomplished. Berger \& Corrsin [2] sought to explain the rise against gravity of particles on the tear film surface via surface tension gradients. They formulated a Lagrangian model for surfactant transport and particle location that explained the role played by surface tension gradients in a short time period following a blink. A typical blink lasts about $1 / 3$ of a second and covers a distance of about 1 cm (Fatt \& Weissman [13]). Wong et al. [40] used a coating flow model to describe tear film formation as the eyelid opens; their model only included capillary and viscous forces and used no slip boundary conditions on both sides of the aqueous layer. Using measured experimental eyelid speed data, they computed a tear film with a thickness of 6 to $8 \mu \mathrm{m}$ over most of the area of the cornea. Their theory may be regarded as the simplest model that satisfactorily explains the process of tear film deposition.

Wong et al. [40] used the same physical assumptions to describe the subsequent drainage of the film. Using a local model at the edge of the tear film, they predicted a power law behaviour that estimated a time of 40s until the film thickness reduced to $800 \mathrm{~nm}$, after which a very rapid rupture process due to van der Waals forces was expected to take over. The average BUT of normal eyes varies according to circumstances, but appears to be in the range of 20-30s (AlAbdulmunem [1]). For dry eyes the BUT may be 10 seconds or less (Bron [7]). We believe that tear film drainage modelling may be improved by examining models of the tear film that include the regions near to the upper and lower eyelids and the film in between: this is the primary purpose of this paper.

Sharma et al. [36] studied the capillarity-driven thinning of the tear film using a lubrication-type model that retained the full curvature terms from the normal stress boundary condition and allowed both no slip or stress free boundary conditions at the deforming free surface. Curvature was specified at the ends of the film. Varying the initial film thickness and curvature at the ends of the film produced a range of plausible BUTs. Amongst other problems, Bertozzi et al. [3] studied the lubrication form of the same capillarity-driven problem as Sharma et al. [36] with a stress-free film surface to examine singularity formation and similarity behaviour. Their results are relevant to the current study and will be dicussed later. Previous models have therefore been largely successful for cases where evaporation is minimal and gravity is absent, so that dry eye is caused by tear film volume deficiency. We wish to explore scenarios when evaporation is suspected of contributing to, or causing, dry eye.

Away from the tear menisci at the eyelids, we shall show that gravity must be accounted for in the description of the tear film. We shall also add evaporation via a "one-sided" approach introduced by Burelbach et al. [9] and Joo et al. 
[17]. The key idea in this model is that all of the parameters (density, thermal conductivity, viscosity) in the vapour are small compared to those in the film and thus the vapour plays a passive role in the evolution of the film. Using this thermal model for evaporation instead of the diffusion limited model discussed in Creech et al. [10], we can make use of experimental data for evaporation rates and illustrate their impact on tear film drainage. Though evaporation on its own is not the primary physical mechanism, we shall show that it can nevertheless work in concert with gravity and capillarity to produce very plausible tear film BUTs.

The paper is organized as follows: the basic model is formulated in section 2 where lubrication theory is applied to determine an evolution equation for tear film thickness. In section 3, numerical results are presented for various combinations of the effects of capillarity, gravity, viscosity and evaporation. The results are discussed in section 4 and conclusions are summarised in section 5 . Finally, some estimates are made in an appendix to show that slip of the mucus layer along the corneal surface is unlikely to be an important effect.

\section{Formulation}

Consider a two-dimensional draining film in a Cartesian coordinate system $\left(x^{\prime}, y^{\prime}\right)$ (a schematic diagram of the coordinate system used is given in figure 1). The corneal surface is assumed to be a vertically-oriented plate positioned at $y^{\prime}=0$; the fact that the plate is considered to be flat is justified because the radius of curvature of the cornea is so much larger than the film thickness (see, for example, Berger \& Corrsin [2]). Gravity acts in the positive $x^{\prime}$-direction so that $\boldsymbol{g}=g \hat{\boldsymbol{i}}$ where $\hat{\boldsymbol{i}}$ is a unit vector in the $x^{\prime}$-direction and $g$ is the acceleration due to gravity. The free surface of the film is denoted by $y^{\prime}=h^{\prime}\left(x^{\prime}, t^{\prime}\right)$. The surface of the mucus layer above the cornea is at $y^{\prime}=0$; it is assumed to be a no-slip surface (though this approximation will be discussed further in the appendix). The half-length of the corneal surface between the two lids after a blink is denoted by $L$. Finally, we assume that the tear film is a linear viscous fluid, and that its density $\rho\left(\mathrm{kg} / \mathrm{m}^{3}\right)$, dynamic viscosity $\mu(\mathrm{Pa} \mathrm{s})$, thermal conductivity $k(\mathrm{~W} / \mathrm{m} / \mathrm{K})$ and specific heat $c_{p}(\mathrm{~J} / \mathrm{kg} / \mathrm{K})$ are all constant.

The equations governing the flow and temperature in the film are

$$
\begin{aligned}
\nabla^{\prime} \cdot \boldsymbol{v}^{\prime} & =0, \\
\rho\left(\boldsymbol{v}_{t^{\prime}}^{\prime}+\left(\boldsymbol{v}^{\prime} \cdot \nabla^{\prime}\right) \boldsymbol{v}^{\prime}\right) & =\mu \nabla^{\prime 2} \boldsymbol{v}^{\prime}-\nabla^{\prime} p^{\prime}+\rho g \hat{\boldsymbol{i}}, \\
\rho c_{p}\left(T_{t^{\prime}}^{\prime}+\left(\boldsymbol{v}^{\prime} \cdot \nabla^{\prime}\right) T^{\prime}\right) & =k \nabla^{\prime 2} T^{\prime},
\end{aligned}
$$

where $t^{\prime}$ denotes time, $\boldsymbol{v}^{\prime}=\left(u^{\prime}, v^{\prime}\right)$ is the fluid velocity, $p^{\prime}$ is the pressure and $T^{\prime}$ is the temperature inside the film. The primes denote dimensional variables.

On $y^{\prime}=0$, we assume that the no-slip condition holds and the temperature is prescribed. Thus

$$
u^{\prime}=v^{\prime}=0, \text { and } T^{\prime}=T_{e y e},
$$


where $T_{\text {eye }}$ is the temperature at the base of the aqueous layer of the tear film which is assumed to be the body temperature of $37^{\circ} \mathrm{C}$. We examine alternative slip conditions in the appendix.

On the free surface $y^{\prime}=h^{\prime}\left(x^{\prime}, t^{\prime}\right)$, we need to specify one kinematic, two dynamic and one thermal boundary condition. These conditions will include the effects of surface tension and evaporation. We therefore set

$$
\begin{aligned}
\frac{\rho\left(v^{\prime}-h_{t^{\prime}}^{\prime}-h_{x^{\prime}}^{\prime} u^{\prime}\right)}{\left(1+h_{x^{\prime}}^{\prime 2}\right)^{1 / 2}} & =J^{\prime}, \\
{\left[\left[\hat{\boldsymbol{n}}^{T} \mathcal{T}^{\prime} \hat{\boldsymbol{n}}\right]\right] } & =H^{\prime} \sigma, \\
\hat{\boldsymbol{t}} \cdot \boldsymbol{v}^{\prime} & =0, \\
{\left[\left[k \hat{\boldsymbol{n}} \cdot \nabla^{\prime} T^{\prime}\right]\right] } & =-L_{m} J^{\prime} .
\end{aligned}
$$

Here $J^{\prime}\left(\mathrm{kg} / \mathrm{sm}^{2}\right)$ is the evaporative mass flux leaving the surface of the film, $\mathcal{T}^{\prime}$ is the stress tensor, $\hat{\boldsymbol{n}}$ and $\hat{\boldsymbol{t}}$ are the unit normal and tangent vectors respectively (with $\hat{\boldsymbol{n}}$ pointing out of the film and $\hat{\boldsymbol{t}} \cdot \hat{\boldsymbol{i}}>0$ ), $\bar{H}$ is the mean curvature of the free surface of the film, defined by

$$
H^{\prime}=\nabla^{\prime} \cdot\left[\left(1+\left|\nabla^{\prime} h^{\prime}\right|^{2}\right)^{-1 / 2} \nabla^{\prime} h^{\prime}\right]
$$

$\sigma(\mathrm{N} / \mathrm{m})$ is the surface tension of the film (assumed constant) and $L_{m}(\mathrm{~J} / \mathrm{kg})$ is the latent heat of vaporisation of the aqueous tear film. The double square brackets denote a jump in the quantity across the free surface; in particular, $[[f]]=f^{f i l m}-f^{\text {air }}$ for the free surface between the film and the ambient air.

The kinematic condition (5) accounts for changes in the shape of the free surface due to evaporation and fluid motion, and (6) accounts for the jump in normal stress at the free boundary due to surface tension (vapour recoil effects may easily be shown to be negligible in this context and have been ignored). The lipid layer above the aqueous layer is modelled by adopting (7), which states that the effect of the lipid layer is to render the free surface tangentially immobile. We expect that this will be accurate once the tear film has been deposited (see, for example Wong et al. [40]). Finally, (8) is a balance between heat flux and latent heat release due to evaporation.

To close the model a constitutive law is required for $J^{\prime}$. Many such laws have been proposed (see, for example Burelbach et al. [9] and Panzarella et al. [27]), but essentially the majority use linearisation to assume that $J^{\prime}$ is proportional to the difference between the saturation temperature $T_{s}(\mathrm{~K})$ in the vapour and the surface temperature $T_{i}^{\prime}=T^{\prime}\left(x^{\prime}, h^{\prime}\left(x^{\prime}, t^{\prime}\right), t^{\prime}\right)$ of the film. We thus write

$$
J^{\prime} \propto\left(T_{i}^{\prime}-T_{s}\right)
$$

where the constant of proportionality will be specified to fit measured evaporation rates in dry eyes.

Finally, we assume that the air above the film is a passive gas that has no 
influence on the flow in the film and that tear film that evaporates into the air is "instantly removed" so that the air above the film has properties that are both spatially and temporally constant. All of the quantities that determine the film flow and evaporation (apart from the vapour density $\rho_{v}$ ) are thus determined from the film itself in this "one-sided" model.

\subsection{Lubrication theory}

We shall now use standard thin-layer theory to model the drainage of the tear film. We begin by non-dimensionalising the equations (1)-(3) and boundary conditions (4)-(8). We set

$$
\begin{gathered}
x^{\prime}=\ell x, y^{\prime}=d y, h^{\prime}=d h, u^{\prime}=U u, v^{\prime}=\epsilon U v, \\
t^{\prime}=\frac{\ell}{U} t, p^{\prime}=\frac{\mu U}{\ell \epsilon^{2}} p, \theta=\frac{T^{\prime}-T_{s}}{T_{\text {eye }}-T_{s}} .
\end{gathered}
$$

where $\epsilon=d / \ell$ and $d$ and $\ell$ are length scales that are to be decided, as is the horizontal velocity scale $U$. The equations become

$$
\begin{aligned}
u_{x}+v_{y} & =0, \\
\epsilon^{2} \operatorname{Re}\left(u_{t}+u u_{x}+v u_{y}\right) & =u_{y y}+\epsilon^{2} u_{x x}-p_{x}+G, \\
\epsilon^{3} \operatorname{Re}\left(v_{t}+u v_{x}+v v_{y}\right) & =\epsilon v_{y y}+\epsilon^{3} v_{x x}-\frac{p_{y}}{\epsilon}, \\
\operatorname{RePr}\left(\theta_{t}+u \theta_{x}+v \theta_{y}\right) & =\theta_{x x}+\frac{1}{\epsilon^{2}} \theta_{y y} .
\end{aligned}
$$

with

$$
u=v=0, \quad \theta=1
$$

on the eye surface $y=0$ and

$$
\begin{aligned}
v-h_{t}-u h_{x} & =\frac{J^{\prime}}{\epsilon \rho U}\left(1+\epsilon^{2} h_{x}^{2}\right)^{1 / 2} \\
-p+\frac{2 \epsilon^{2}\left[v_{y}-u_{y} h_{x}+\epsilon^{2}\left(u_{x} h_{x}^{2}-v_{x} h_{x}\right)\right]}{1+\epsilon^{2} h_{x}^{2}} & =\frac{\sigma \epsilon^{3} h_{x x}}{\mu U\left(1+\epsilon^{2} h_{x}^{2}\right)^{1 / 2}} \\
u+\epsilon^{2} v h_{x} & =0 \\
\theta_{y}-\epsilon^{2} h_{x} \theta_{x} & =-\frac{\epsilon \ell L_{m} J^{\prime}}{k\left(T_{\text {eye }}-T_{s}\right)}\left(1+\epsilon^{2} h_{x}^{2}\right)^{1 / 2}
\end{aligned}
$$

on $y=h(x, t)$. Here

$$
\operatorname{Re}=\frac{\ell U}{\nu}, \quad \operatorname{Pr}=\frac{\mu c_{p}}{k}, \quad G=\frac{\rho g d^{2}}{\mu U} .
$$

we shall now estimate some of the terms in the equations and boundary conditions. We assume that $d=10 \mu \mathrm{m}$ (as observed in section 1 , this may be regarded 
as a typical tear film thickness). We further assume that $\ell$ denotes the meniscus length. This is given (see, for example, Wilson [39]) by

$$
\ell=d^{1 / 3} D^{2 / 3}
$$

where $D=\sqrt{\sigma / \rho g}$ is the static meniscus radius. Using $g=9.81 \mathrm{~m} / \mathrm{s}^{2}, \rho=$ $10^{3} \mathrm{~kg} / \mathrm{m}^{3}$ and $\sigma=0.045 \mathrm{~N} / \mathrm{m}$ (Wong et al. [40], Miller [21]), we find that $D=2.1 \mathrm{~mm}$ and $\ell=0.36 \mathrm{~mm}$. A typical draining velocity may be estimated by assuming that the film drains $1 \mathrm{~cm}$ in around 10s; thus $U \sim 10^{-3} \mathrm{~m} / \mathrm{s}$. With $\mu=1.3 \times 10^{-3} \mathrm{~Pa}$ s (Ehlers [12], Wong et al. [40]) we now find that

$$
G \sim 0.75
$$

and we conclude that gravity must be retained in the equations. Since $G$ is order unity, we further infer that the correct scale for the velocity is

$$
U=\frac{\rho g d^{2}}{\mu} \sim 0.75 \mathrm{~mm} / \mathrm{s},
$$

which is used henceforth. Some other parameters may now be estimated: we have

$$
\epsilon \sim 0.028, \quad \operatorname{Re} \sim 0.2, \quad \epsilon^{2} \operatorname{Re} \sim 1.6 \times 10^{-4}
$$

and, with $k=0.68 \mathrm{~W} / \mathrm{mK}$ and $c_{p}=4.19 \times 10^{3} \mathrm{~J} / \mathrm{kg} \mathrm{K}$ (values for water)

$$
\epsilon^{2} \operatorname{RePr} \sim 1.3 \times 10^{-3} .
$$

Finally, we note that from our choice of scalings $\epsilon^{3} \sigma /(\mu U)=1$ so that the term on the right hand side of (19) must be retained.

The leading-order equations and boundary conditions are therefore

$$
\begin{aligned}
u_{x}+v_{y} & =0 \\
u_{y y}-p_{x}+1 & =0 \\
p_{y} & =0 \\
\theta_{y y} & =0
\end{aligned}
$$

with

$$
u=v=0, \quad \theta=1
$$

on $y=0$ and

$$
\begin{aligned}
v-h_{t}-u h_{x} & =\frac{J^{\prime} \ell \mu}{d^{3} \rho^{2} g} \\
-p & =h_{x x} \\
u & =0 \\
\theta_{y} & =-\frac{\epsilon \ell L_{m} J^{\prime}}{k\left(T_{\text {eye }}-T_{s}\right)}
\end{aligned}
$$


on $y=h(x, t)$. We now need to determine the importance of surface tension and evaporation. The non-dimensional parameter that determines the importance of surface tension is unity by dint of our definition of $\ell$, but we note that, away from the menisci near to the centre of the tear film, we anticipate a slowly-varying film thickness with $h$ nearly constant. The effects of capillarity are thus confined to regions of the tear film near the menisci where $h_{x x} \sim 1$.

Finally, the effects of evaporation are governed by two non-dimensional parameters. With $L_{m}=2.3 \times 10^{6} \mathrm{~J} / \mathrm{kg}$ (the value for pure water), $T_{\text {eye }}-T_{s}=1 \mathrm{~K}$ and $J^{\prime}=3 \times 10^{-5} \mathrm{~kg} / \mathrm{m}^{2} / \mathrm{s}$ (a value for mildly dry eyes), we find that

$$
\frac{J^{\prime} \ell \mu}{d^{3} \rho^{2} g} \sim 1.4 \times 10^{-3}, \quad \frac{\epsilon \ell L_{m} J^{\prime}}{k\left(T_{\text {eye }}-T_{s}\right)} \sim 1.0 \times 10^{-3} .
$$

Although for much of the film draining process evaporation is therefore insignificant, we note that when the film becomes thin (for example when $d \sim 1 \mu \mathrm{m}$ ) evaporation may have a much more important role to play in the final rupture of the tear film. We thus retain the evaporation terms in the model.

Solving for the velocity components and the pressure in the usual way now gives that $p=-h_{x x}$ and

$$
u=\frac{y(y-h)\left(p_{x}-1\right)}{2}, \quad v=\frac{y^{2}}{4} h_{x}\left(p_{x}-1\right)-\frac{1}{2} p_{x x}\left(\frac{y^{3}}{3}-\frac{h y^{2}}{2}\right),
$$

and imposing the free surface condition now yields

$$
h_{t}+\left[\frac{h^{3}}{12}\left(h_{x x x}+1\right)\right]_{x}+\frac{\ell \mu J^{\prime}}{d^{3} g \rho^{2}}=0 .
$$

We now non-dimensionalise the evaporation term by setting $J^{\prime}=k J /\left(d L_{m}\right)$ and assuming that $J=J_{0}\left(T^{\prime}\left(h^{\prime}\right)-T_{s}\right)$ where $J_{0}$ is dimensionless. The problem for the temperature becomes

$$
\theta_{y y}=0, \quad \theta(x, 0, t)=1, \quad \theta_{y}(x, h, t)=-J_{0} \theta(x, h, t)
$$

and thus

$$
\theta=1-\frac{J_{0} y}{1+J_{0} h}
$$

The governing equation for the evolution of the film thickness is thus

$$
h_{t}+\frac{E}{J_{0}^{-1}+h}+\left[\frac{h^{3}}{12}\left(h_{x x x}+G\right)\right]_{x}=0 .
$$

where

$$
E=\frac{k\left(T_{\text {eye }}-T_{s}\right) \ell \mu}{d^{4} g \rho^{2} L_{m}} .
$$

This equation is similar to those derived by Burelbach et al. [9] and Panzarella 
et al. [27]. We further note that, by our choice of scalings, $G=1$; however, we have retained $G$ in (42) to allow us to examine cases without gravity by setting $G=0$.

The equation (42) requires one initial and four boundary conditions. We shall assume that

$$
h( \pm L)=h_{0},
$$

where $L$ is the half-length of the film. This is tantamount to assuming that the tear film is "pinned" at the top and bottom lids. It is generally accepted (see, for example Fatt \& Weissman [13]) that material above the "gray line" on the superior and inferior tarsi is unwettable.

Two other boundary conditions are required, and sound cases may be made for a number of different choices. First, the tear film curvatures $h_{x x}$ at each end of the film may be specified. In the absence of the effects of gravity, the static meniscus has a constant curvature; even with the effects of gravity present, however, many sources have suggested that constant curvature menisci have been observed. Second, the slope $h_{x}$ at either end may be specified; this specifies the contact angle. A constant contact angle has frequently been assumed in the fluid mechanics literature, but there seem to be few direct measurements of this quantity for eyes. Finally, it is also possible to specify the fluxes at either end. All of these three possibilities are investigated below, though when we have specified the fluxes we have assumed that they are zero; results including the effects of tear film production and elimination will be postponed to a future study.

The initial conditions for which we compute solutions assume a flat film with parabolic ends, namely,

$$
h=\left\{\begin{array}{ll}
h_{\min }(0), & |x|<L-\Delta x_{m} \\
h_{\min }(0)+\Delta h_{m}\left[|x|-\left(L-\Delta x_{m}\right)\right]^{2}, & |x|>L-\Delta x_{m} .
\end{array} .\right.
$$

Here $\Delta x_{m}$ is the width of the parabolic initial meniscus; $h_{\min }(0)=1$ (corresponding to an initial thickness of $10 \mu \mathrm{m}$ ) is used for all computations that follow. We shall vary $\Delta h_{m}$ and $\Delta x_{m}$ because these can vary from person to person, but all values used will be averages based on experimental evidence. Note that $h_{0}=h_{\min }(0)+\Delta h_{m}\left(\Delta x_{m}\right)^{2}$ for this choice of initial condition. (For normal eyes, typically $1 \leq \Delta x_{m} \leq 2$ when measured in units of $\ell$ and $h_{0} \sim \ell$, while for dry eyes $\Delta x_{m}<1$ and $h_{0}<\ell$.)

\subsection{Equilibrium solutions}

Equation (42) is nonlinear and, in general, must be solved numerically: this will be addressed in the next section. First, however, we briefly examine some of its properties.

Equilibrium (i.e. time-independent) solutions may exist in the absence of gravity and evaporation. Such solutions satisfy

$$
\left(\frac{h^{3}}{12} h_{x x x}\right)_{x}=0
$$


For cases where there is no flow in the film, the equation may be integrated to yield $h_{x x x}=0$, which implies possible parabolic equilibria. Whether or not such equilibria exist depends upon the boundary conditions; in all cases that we consider, the film thickness is specified at the ends so that $h( \pm L)=h_{0}$. When no flux conditions are applied at the ends of the film so that $h_{x x x}( \pm L)=0$, solutions now take the form

$$
h=h_{0}+\frac{C}{2}\left(x^{2}-L^{2}\right)
$$

where the constant $C$ may be determined by integrating $h$ and equating the result to a prescribed total area $A_{0}$ of the film. We obtain

$$
C=\frac{6 L h_{0}-3 A_{0}}{2 L^{2}}
$$

For sufficiently small values of $A_{0}$, we find that $h$ is non-positive. This first occurs when $A_{0}=2 L h_{0} / 3$ and thus $C=2 h_{0} / L^{2}$; in that case, $h(0)=0$ and when $A_{0}$ is less than or equal to this value, no physically sensible equilibrium solution is possible. For sufficiently large $A_{0}$, equilibria always exist.

The case when the curvature $h_{x x}$ is specified at the ends (say $h_{x x}( \pm L)=r$ ), was studied by Bertozzi et al. [3]. With the scalings defined in section 2, equilibria are given as by (47) with $C=r$. If $r<2 h_{0} / L^{2}$ then equilibria exist; for larger $r$, there are no equilibria. The case when the contact slope $h_{x}$ is specified is similar. For $h_{x}( \pm L, t)= \pm m$, we have $C=m / L$ and if $m<2 h_{0} / L$ there are equilibria; for larger $m$, there are none.

When the effects of gravity are included, matters are complicated slightly. Equilibrium solutions with no flux must now satisfy

$$
h_{x x x}+1=0 .
$$

The solution to this equation is a cubic polynomial in $x$ containing three arbitrary constants. Two of these may be determined by imposing $h( \pm L)=h_{0}$, and, as in the case with no gravity, the third may be determined if the total area $A_{0}$ of the film is known. We obtain

$$
h=h_{0}+\frac{\left(x^{2}-L^{2}\right)}{12 L^{3}}\left(18 h_{0} L-2 L^{3} x-9 A_{0}\right)
$$

Clearly for fixed $h_{0}$ and $A_{0}$ this solution becomes non-positive as $L$ increases. For representative values of $h_{0}=17$ (corresponding to a meniscus height $0.17 \mathrm{~mm}$ ) and $L=14$ (an inter-lid distance of just over $1 \mathrm{~cm}$ ) we find that there are no equilibrium solutions unless $A_{0}>6600$. Since a typical initial profile (45) (for example with $L=14, \Delta x_{m}=2$ and $\Delta h_{m}=4$ so that $h_{0}=17$ ) has non-dimensional area 49.3, we conclude that normal eyes contain far too little tear film to allow equilibrium tear film shapes.

Finally, when both evaporation and gravity are included we expect on physical grounds that equilibrium solutions may be possible if there is a net inflow into the tear film region to balance the mass loss due to evaporation. Rigorous analysis of the boundary value problem appears to be challenging in this case, however. 


\subsection{Film rupture}

It is also worth noting that, in the absence of evaporative losses and gravitational effects, it has long been known that solutions to (42) that are initially everywhere positive cannot ever become zero provided $h_{x x}$ remains bounded (see, for example Bertozzi et al. [3]). It seems plausible that a similar result holds when gravity is included. When the evaporation terms are included it is clear that film rupture can take place, however. Although evaporation is obviously a key component of our model, it should be remembered that other effects may be important for very thin films. For example, van der Waals forces may be important in the final details of film rupture (see, for example, Burelbach et al. [9], Sharma et al. [34]). We have chosen to ignore such short-range forces in our model, however.

\section{Results}

To generate detailed results showing the effects of surface tension, gravity and evaporation, the nonlinear PDE (42) for the tear film thickness was integrated numerically. The spatial derivatives were discretized using a conservative finite difference scheme and the resulting ODEs for the $h_{j}(t)=h\left(x_{j}, t\right)$ were solved using DASPK (Brenan et al. [6]). DAsPK uses variable BDF (Backward Differentiation Formula) time stepping which is stable and efficient for this type of problem; this type of approach has previously been used successfully in similar circumstances (Braun et al. [5], Naire et al. [25]). The conservative finite differencing scheme is essential for the extended times for which we compute solutions. 4000 uniformly spaced grid points were used for all results presented here; tests showed that increasing the number of grid points further made essentially no difference to the results.

Table 1 gives the values of the parameters used in the computations. Unless otherwise noted, all computations were performed on a domain $-L \leq x \leq L$ with $L=14$; this corresponds to a dimensional distance between the eyelids of $1.008 \mathrm{~cm}$.

\subsection{Capillarity only}

Solutions were first computed in the absence of gravitational and evaporative effects $\left(G=J_{0}=E=0\right)$ in order to completely validate the numerical code and to compare with previous studies.

Results for the initial condition $\Delta h_{m}=\Delta x_{m}=2$ (corresponding to a realistic, but rather small meniscus height and curvature) with the tear film curvature specified at $x= \pm L$ are shown in figure 2 . Here and henceforth, a nondimensional time of 2.08 corresponds to 1 second. We observe that the thickness of the film changes most rapidly near to the edges of the domain; the minimum film thickness,

$$
h_{\min }(t)=\min _{-L \leq x \leq L} h(x, t)
$$


Table 1: Unless otherwise stated, these properties and dimensional quantities were used in all of the computations reported below. The value of $J^{\prime}$ is from Mathers [19] and is the highest average for dry eye patients; the values of $\Delta T=T_{\text {eye }}-T_{s}, J_{0}$ and $E$ recover this dimensional value when $\theta=1$.

\begin{tabular}{|c|c|}
\hline Parameter & Value \\
\hline$\rho$ & $10^{3} \mathrm{~kg} / \mathrm{m}^{3}$ \\
$g$ & $9.81 \mathrm{~m} / \mathrm{s}^{2}$ \\
$\mu$ & $1.3 \times 10^{-3} \mathrm{Pas}$ \\
$\sigma$ & $0.045 \mathrm{~N} / \mathrm{m}$ \\
$L_{m}$ & $2.3 \times 10^{6} \mathrm{~J} / \mathrm{kg}$ \\
$k$ & $0.68 \mathrm{~W} / \mathrm{m} / \mathrm{K}$ \\
$\Delta T=T_{\text {eye }}-T_{s}$ & $10 \mathrm{~K}$ \\
$J^{\prime}$ & $6 \times 10^{-5} \mathrm{~kg} / \mathrm{m}^{2} / \mathrm{s}$ \\
$d$ & $10^{-5} \mathrm{~m}$ \\
$\ell$ & $3.6 \times 10^{-4} \mathrm{~m}$ \\
$U$ & $7.5 \times 10^{-4} \mathrm{~m} / \mathrm{s}$ \\
$\epsilon$ & 0.028 \\
$E$ & 14.1 or 0 \\
$J_{0}^{-1}$ & 4930 or $\infty$ \\
$G$ & 0 or 1 \\
\hline \multicolumn{2}{|c}{}
\end{tabular}

occurs near the edge of the meniscus at about $x=12$ as found in Wong et al. [40]. The film curvature (shown in figure 3) is non-zero everywhere and thus flow occurs over all of the tear film, though in the interior of the film away from the menisci both the curvature and flow speeds are small. As the film thins near to the menisci, it also thickens in its interior (though for very large time scales, the centre of the film does eventually thin). However, the results of figure 3 show that no thickening of note occurs until at least $t=512(246 \mathrm{~s})$, which is a great deal later than the BUT found experimentally by Al-Abdulmunem [1].

When $h_{x x}( \pm L, t)$ is specified, the meniscus shape at the ends of the tear film is prescribed. However, different boundary conditions were also considered. Results using a no flux condition, which, with $G=0$, is $h_{x x x}( \pm L, t)=0$, are shown in figures 4 and 5 . We find that the thinning of the film is slowed compared to the case of figure 2 with $h_{x x}( \pm L, t)$ specified. For example, for the case with initial conditions $\Delta h_{m}=\Delta x_{m}=2$, we have $h_{\min }(512) \approx 0.126$ for the no flux case of figures 4 and 5 . In contrast, for the case with $h_{x x}( \pm L, t)$ given (shown in figures 2 and 3$)$, we have $h_{\min }(512) \approx 0.054$. We shall discuss this point further below. The curvature of the meniscus, shown in figure 5 , steadily decreases at the end of the film for the no-flux case as liquid accumulates there.

When $h_{x}( \pm L, t)$ is specified, the results are very similar to those obtained by specifying the curvature, so long as the meniscus height is relatively large compared to the film thickness $\left(\Delta h_{m} \geq 2\right)$. Since this is the parameter range of interest, we have omitted these results.

When only capillarity is active, the BUT may be defined as the time required for $h_{\min }(t)$ to reach $750 \mathrm{~nm}$, after which we assume that van der Waals forces would cause rupture as in Wong et al. [40]; we believe this thickness to be 
generous for these forces to take over. Using this criterion, for a film with $\Delta h_{m}=$ $\Delta x_{m}=2$, the nondimensional time to reach $0.75 \mu \mathrm{m}$ thickness is 256 , or just over 2 minutes. However, for a film with $\Delta h_{m}=8, \Delta x_{m}=2$, the BUT reduces greatly to 7.7 seconds. For the same film but with a smaller cut-off criterion of say $0.54 \mu \mathrm{m}$, we find that $h_{\min }(32)=0.54 \mu \mathrm{m}$ (about 15.4 seconds). The BUT is thus sensitive both to the initial conditions and to the assumed cut-off thickness for rupture to begin. A typical thickness for van der Waals forces to become important and thus for the rupture to begin for an aqueous film is order $0.1 \mu \mathrm{m}$ (see, for example, Sharma \& Ruckenstein [35]).

Taking the view that the cornea is easily wettable, Sharma et al. [36] concluded that rupture never actually occurred; when the film thickness is reduced to about $0.1 \mu \mathrm{m}$, a stable film should be formed. We take the view that break-up can occur when evaporation is included in the model, and for convenience we retain this view in all the cases that we discuss below.

Varying the size of the meniscus clearly has a substantial effect on the evolution of $h_{\text {min }}(t)$. This agrees with the results of Sharma et al. [36]. Results for the evolution of $h_{\min }(t)$ for several initial conditions and both no flux and fixed curvature boundary conditions are shown in figure 6 . As the curvature at the boundary increases, it becomes dominant in determining the thinning behaviour because the no flux solution tends to the constant curvature behaviour. It may be confirmed that the minimum film thickness evolves like $h_{\min }(t) \propto t^{-0.46}$ for large times as found by Wong et al. [40]. Our result, however, has been computed using a global model for film thickness. Similar results were obtained by Sharma et al. [36] using a model for the whole tear film.

Bertozzi et al. [3] found $t^{-0.5}$ behaviour for the infinite-time film rupture singularity, obtaining both analytical and numerical results, for a problem identical to that studied in this section (though with a different initial condition). Their adaptive mesh code clearly identified this behaviour for a number of decades below $h=0.01$, which is less than the thickness for which we can compute reliable numerical solutions. For very large times, we therefore expect that our exponent of -0.46 would be modified to -0.5 .

\subsection{Capillarity and evaporation only}

We now consider cases where $G=0$ but $E$ and $J_{0}$ are non-zero, so that evaporation is active. Numerical results for a film with curvature specified at the ends are given in figure 7 . The film remains symmetric about the origin, and the evolution is in many ways similar to the preceeding case. However, evaporation now causes the film to thin everywhere (except at the pinned ends), leading to a finite BUT of roughly $82(39.4 \mathrm{~s})$. For a case with $\Delta h_{m}=8$ (which is more representative of the value that might be expected for a normal eye), the BUT is $43.5(21 \mathrm{~s})$. When evaporation is included, the film reaches zero thickness in finite time: hence no cut-off value is required or used here. Further criteria for rupture will be considered below. 
Figure 8 shows a comparison of results for $h_{\min }(t)$ for four different initial conditions with and without evaporation. The ostensibly small rate of evaporation eventually dominates the capillarity-driven thinning noted in the previous section (where the rate of thinning decreases with time). This is shown by the departure of the curves with evaporation from the those without at later times and the subsequent rupture of the film. This figure illustrates how even a relatively small evaporation rate can affect the later stages of tear film drainage; this result is central to our work.

\subsection{Capillarity and gravity only}

Next we study tear film evolution with $G=1$ and $E=J_{0}=0$, so that gravity now acts in the problem, but evaporation is absent.

Results for initial conditions $\Delta h_{m}=1, \Delta x_{m}=2$ (a relatively small meniscus) and boundary conditions $h_{x x}( \pm L, t)=1$ are shown in figures 9 and 10. The inclusion of gravity causes the region near to the upper lid to thin more than the the region near to the lower lid. At the lower lid, a shape that at each time is close to that of a static meniscus develops; this arises essentially from a balance between gravitational and capillary forces. Clear qualitative differences in the draining process are observed when gravity is included; the film is no longer symmetric about $x=0$ and away from the menisci gravity rather than capillarity dominates the drainage process. Gravity also causes the formation of a bulge similar to a classical "tear pool" near to the lower lid.

Results at $t=64(31 \mathrm{~s})$ for no flux boundary conditions and four different initial meniscus conditions are shown in figure 11. We observe that, in all four cases, the film appears to consist of four distinct regions: (i) near to $x=-L$ the film thickness decreases sharply as gravity drains fluid away from the region near to the upper lid, (ii) the film thickness slowly increases in a gravity-dominated region that extends over the middle part of the tear film, (iii) as the lower lid is approached, capillary, viscous and gravity effects all become important and a capillary wave is formed, (iv) the capillary wave is connected to the tear pool meniscus near to the lower lid. The qualitative details of the film drainage are therefore relatively insensitive to the initial conditions.

In figure 12 the minimum film thickness $h_{\min }(t)$ is plotted for a case with no flux boundary conditions for four different values of the meniscus parameter $\Delta h_{m}$. In order to illustrate the differences between the thinning process near to the top and the bottom of the tear film, both of the local minima near the top and the bottom of the film are plotted. We observe that the $h_{\min }$ changes a great deal more rapidly near the top of the eye. These results may also be used to show that, near to the top lid, $h_{\min }(t) \sim t^{-0.8}$ for large times. This result may be contrasted with the case where no gravity is included where $h_{\min }(t) \sim t^{-0.46}$, and clearly shows that gravity has a non-trivial role to play in the analysis of tear film drainage and should not be ignored.

It is also possible to investigate the effects of different boundary conditions 
on the evolution of the film. Wong et al. [40] used constant curvature conditions consistent with their view that tear film deposition was essentially a coating flow problem. As noted above, one could just as well apply fixed slope (i.e. contact angle) or no flux conditions. When gravity is included, the no flux condition becomes $h_{x x x}( \pm L, t)+G=0$. We have investigated all three of these cases, though space does not permit a detailed analysis of all the results. However, fixing either $h_{x}$ or $h_{x x}$ at the ends yields very similar results to those discussed above when the meniscus height at the ends is large compared to $h_{\min }(0)$; either case causes faster drainage than cases where no flux boundary conditions are applied, though the behaviour may be more complicated for smaller menisci.

\subsection{Capillarity, gravity and evaporation}

Finally, results were computed for cases when both gravity and evaporation were active $\left(G=1, E>0, J_{0}>0\right)$. In most respects, the evolution of the tear film is similar to that observed in the preceeding section, however, when evaporation is included rupture again occurs in finite time. As we have already observed, evaporation becomes increasingly important as the film thins. For brevity, we simply compare results at time $t=64(31 \mathrm{~s})$ for $\Delta h_{m}=\Delta x_{m}=2, L=14$ and constant curvature boundary conditions, with and without both gravity and evaporation. The results plotted in figure 13 show that evaporation reduces the film thickness, but that the thickness change is not dramatic. However, when the thickness decrease is slowing down as in either case without evaporation, the relative importance of evaporation increases as a means of thinning the tear film.

Finally, figure 14 shows the evolution of $h_{\min }(t)$ using parameters $\Delta h_{m}=8$, $\Delta x_{m}=2$ and $L=14$ and fixed curvature boundary conditions. These may be regarded as our "best guess" at parameters for eyes with a normal tear film volume, but dry eye evaporation rates. Both gravity and evaporation clearly enhance drainage and when both are included rupture occurs at a much reduced time of about $t=25.5(12 \mathrm{~s})$.

\section{Summary and discussion}

When the effects of capillarity alone are present, our model is closely related to that studied by Bertozzi et al. [3] and Sharma et al. [36], though the latter do not adhere strictly to lubrication theory. Both of these studies also use different initial conditions. The power law exponent of Wong et al. [40], who carried out a local analysis, was recovered.

Including evaporation significantly reduced the tear film break-up time (BUT). The essentially linear rate of thinning due to evaporation becomes significant at large enough times because the power law thinning from the capillarity slows as time increases and the film thins. Figure 8 clearly illustrates this behaviour.

Including gravity clearly shows the tear film is redistributed from the top to 
the bottom of the eye; the accumulation is more noticeable for small menisci, than when larger (and possibly more realistic) menisci are used. Flow in the extensive, relatively flat, middle of the film is dominated by gravity; similar behaviour has been observed for free films (Braun et al. [5]; Naire et al. [25]; Schwartz \& Roy [31]). The inclusion of gravity promotes film break-up nearer to the top of the film and may decrease the exponent in the thinning of the film there. The change in the exponent is significant, the behaviour changing from $t^{-0.46}$ to $t^{-0.8}$ over the time range of interest. When both gravity and evaporation are included, obviously the break-up of the film near to the upper lid occurs even more quickly.

Whichever effects are included, we observe that, when the initial curvature in the menisci is made large enough, the upper meniscus behaviour becomes relatively insensitive to the type of boundary condition used. Though the meniscus curvatures used in this study were chosen to reflect average values for human eyes, they are still smaller than some values pertaining to patients with dry eye due to insufficient tear production; using such values would invalidate the assumptions of lubrication theory. Although the most extreme meniscus conditions that we have computed stretch the validity of lubrication theory almost to its limits, we believe that the trends have been correctly identified in this study.

Table 2: Summary of non-dimensional Break-Up Times (BUTs) (and BUTs in seconds) with $h_{x x}$ specified at the boundaries. The addition of gravity and evaporation reduces BUTs. The case with infinite BUT occurs because there is a net influx of fluid at the top of the film for this set of parameters.

\begin{tabular}{|c|c|c|c|c|c|}
\hline & & BUT & BUT & BUT & BUT \\
$\Delta h_{m}$ & $h_{x x}( \pm L, t)$ & $G=0$ & $G=0$ & $G=0$ & $G=1$ \\
& & $E=0$ & $E=14.1$ & $E=14.1$ & $E=14.1$ \\
& & $J_{0}=0$ & $J_{0}^{-1}=4930$ & $J_{0}^{-1}=4930$ & $J_{0}^{-1}=4930$ \\
& & $h_{m i n}=0.05$ & $h_{m i n}=0.05$ & $h_{\min }=0$ & $h_{\min }=0$ \\
\hline 1 & 2 & $2180(1048 \mathrm{~s})$ & $104(50 \mathrm{~s})$ & $121(58 \mathrm{~s})$ & $\infty$ \\
2 & 4 & $627(301 \mathrm{~s})$ & $64.2(31 \mathrm{~s})$ & $82(39 \mathrm{~s})$ & $104.8(50 \mathrm{~s})$ \\
4 & 8 & $153(74 \mathrm{~s})$ & $38.1(18 \mathrm{~s})$ & $57.5(28 \mathrm{~s})$ & $42.8(21 \mathrm{~s})$ \\
8 & 16 & $38.0(18 \mathrm{~s})$ & $19.7(10 \mathrm{~s})$ & $43.5(21 \mathrm{~s})$ & $25.5(12 \mathrm{~s})$ \\
\hline
\end{tabular}

Computed BUTs are summarised in Table 2 for cases where the curvature $h_{x x}$ is specified at the boundary. The addition of both gravity and evaporation speeds up the rupture process. The notable exception to this is the case with the smallest meniscus when gravity is included; here, an influx occurs at the upper boundary, the film reaches a steady state profile, and the film never ruptures.

When the effects of gravity are included, film rupture always occurs near to the upper lid. Without gravity all previous theoretical models show no preference for either the top or the bottom of the film as far as film rupture is concerned, though this may be introduced by choosing where to compare with experimental data 
(Creech et al. [10]). Recent experimental results Bitton \& Lovasik [4] suggest that film break-up occurs preferentially near to the bottom and side quadrants of the cornea, and occurs less frequently near to the top of the cornea. This highlights a shortcoming of our model, for three dimensional flow effects, or conditions on the surface of the cornea or conjunctiva may play an important role in determining film break-up location (Sharma [33]).

\section{Conclusions}

Evaporation can dramatically shorten the life of a vertical film model of a human tear film even when the evaporation rate appears to be small. This is because the capillarity-driven thinning of the meniscus slows down (according to a power law) as time increases, allowing evaporation to become significant when the film is thinned.

We have also shown that gravity dominates the evolution away from the menisci at either eyelid. Gravity also speeds up the thinning at the top of the film in the cases that we studied. Since BUT tests are normally carried out with the subject's head held upright assuming a horizontal gaze, the model indicates a preference for tear film break-up at the top of the film, near the upper eyelid.

Our model is a two-dimensional one, and significant three dimensional flow effects occur in real eyes. The aqueous tear supply enters at from the lacrimal glands at the top, outer part of the eye, and the tear film drains out at the bottom of the eye near the nose through the puncta. The boundary conditions applied in this and other two-dimensional models are therefore caricatures of what really happens near to the eyelid. Further three-dimensional flow modelling would be extremely valuable.

Another significant step forward would be to develop a model that allows a mobile aqueous film surface and surfactant transport on its surface; it may then be possible to develop a unified model of film formation and drainage. We are currently investigating such models.

\section{Acknowledgements}

The authors would like to thank Mr. C. Canning (Eye Unit, Southampton General Hospital, Tremona Road, Southampton) and Dr. J.N. Dewynne (OCIAM, Oxford University) for helpful conversations. RJB is grateful for support from EPSRC grant GR/R90666/01 and NSF Grant DMS 96-31287.

\section{Appendix A: Slip coefficient}

In this appendix we estimate the possible effect of a mucus layer that adheres to the surface of the corneal epithelium, thus allowing an aqueous tear film layer above it to "slip". 
Sharma et al. [34] developed a physical analogue system to represent the aqueous and mucus layers in the tear film. Their study suggests that, under some circumstances, the mucus layer may indeed cause slip for the aqueous layer, thus altering the no-slip boundary conditions (4) that were used in our model above. We shall investigate slip in the context of gravitational drainage.

Consider a case similar to that shown in figure 1 where a Newtonian viscous aqueous tear film layer of constant thickness $h_{a}$ overlays a Newtonian viscous mucus layer $-h_{m}<y<0$. We assume that the mucus layer does not slip on the flat solid surface $y=-h_{m}$ and that the aqueous layer is tangentially immobile at $y=h_{a}$. We also assume that both layers are flat, that there is no applied pressure gradient, and that the mucus layer is about ten times more viscous than and one tenth the thickness of the aqueous layer (Sharma et al. [34]). Using dimensional variables throughout and identifying quantities in the mucus and aqueous layers using subscripts $m$ and $a$ respectively, we have

$$
\begin{aligned}
\mu_{a} u_{a y y}+\rho_{a} g & =0 & & \left(0<y<h_{a}\right) \\
\mu_{m} u_{m y y}+\rho_{m} g & =0 & & \left(-h_{m}<y<0\right)
\end{aligned}
$$

with boundary conditions

$$
u_{m}\left(-h_{m}\right)=0, \quad u_{a}\left(h_{a}\right)=0
$$

and interfacial conditions

$$
u_{m}(0)=u_{a}(0), \quad \mu_{m} u_{m y}(0)=\mu_{a} u_{a y}(0)
$$

Solving (51)-(54), we find that

$$
u_{a}=-\frac{g \rho_{a} y^{2}}{2 \mu_{a}}+A_{a} y+B_{a}, \quad u_{m}=-\frac{g \rho_{m} y^{2}}{2 \mu_{m}}+A_{m} y+B_{m}
$$

where the constants $A_{a}, A_{m}, B_{a}$ and $B_{m}$ are given by

$$
\begin{gathered}
A_{a}=-\frac{g\left(\mu_{a} \rho_{m} h_{m}^{2}-\mu_{m} \rho_{a} h_{a}^{2}\right)}{2 \mu_{a}\left(h_{m} \mu_{a}+h_{a} \mu_{m}\right)}, \\
A_{m}=-\frac{g\left(\mu_{a} \rho_{m} h_{m}^{2}-\mu_{m} \rho_{a} h_{a}^{2}\right)}{2 \mu_{m}\left(h_{m} \mu_{a}+h_{a} \mu_{m}\right)}, \\
B_{a}=B_{m}=\frac{g h_{a} h_{m}\left(\rho_{a} h_{a}+\rho_{m} h_{m}\right)}{2\left(h_{m} \mu_{a}+h_{a} \mu_{m}\right)},
\end{gathered}
$$

Let us now define a slip coefficient $\lambda$ in the standard manner by assuming that $\lambda u_{a y}(0)=u_{a}(0)$. This gives

$$
\lambda=\frac{B_{a}}{A_{a}}=\frac{h_{m}\left(1+\frac{\rho_{m} h_{m}}{\rho_{a} h_{a}}\right)}{\frac{\mu_{m}}{\mu_{a}}-\frac{\rho_{m}}{\rho_{a}} \frac{h_{m}^{2}}{h_{a}^{2}}} .
$$


To estimate $\lambda$ we now assume that $\rho_{a}=\rho_{m}, \mu_{m}=10 \mu_{a}, h_{a}=10 h_{m}$ and $h_{a}=10 \mu \mathrm{m}$. This gives $\lambda \sim 10^{-7} \mathrm{~m}$. The relative importance of slip is measured by the ratio $R=\lambda / y$, and, using the $y$-scale of $h_{a}=d=10 \mu \mathrm{m}$ introduced in the main body of the text, we find that

$$
R \sim 10^{-2}
$$

This suggests that including slip arising from the mucus layer is likely to affect the results by only about one per cent. Sharma et al. [34] suggested that a mucus/aqueous viscosity factor of 10 was probably a rather low estimate for a quantity that might be as high as 100 . If this were true, however, the slip coefficient would decrease even more. The only circumstances in which mucus/aqueous slip might become important are therefore (a) if the viscosity factor was, in reality, less than 10, or (b) if the mucus layer was a great deal thicker than $1 \mu \mathrm{m}$. Although there therefore seems to be no possibility of slip being important for the analysis of tear film drainage in human eyes, it may have to be included for some animal eyes (for example, it has been claimed that the mucus layer in a rabbit eye may be as thick as $30 \mu \mathrm{m})$.

Finally, we note that it is also claimed in Sharma et al. [34] that the mucus layer may slip along the surface of the cornea; we have not investigated the consequences of this possibility. It seems likely, however, that even if the mucus layer does slip, its motion is likely to be on a significantly slower time scale than the drainage of the aqueous film.

\section{References}

1. Al-Abdulmunem, M.A., Tear film break-up time in normal Saudi Arabian subjects, ICLC, (1997). 24, 145-147.

2. Berger, R.E., Corrsin, S., A surface tension gradient mechanism for driving the pre-corneal tear film after a blink, J. Biomechanics, (1974). 7, 225238.

3. Bertozzi, A.L., Brenner, M.P., Dupont, T.F., Kadanoff, L.P., Singularities and Similarities in Interface Flows, In: Trends and Perspectives in Applied Mathematics 155-208, Sirovich, L. (ed.), (1994). New York: SpringerVerlag,

4. Bitton, E., Lovasik, J.V., Longitudinal analysis of precorneal tear film rupture patterns, Lacrimal Gland, Tear Film and Dry Eye Syndromes 2, Sullivan, D.A., Dartt, D.A. \& Meneray, M.A. (eds.), (1998). New York: Plenum Press,

5. Braun, R.J., Snow, S.A., Pernisz, U.C., Gravitational drainage of a tangentially-immobile thick film., J. Colloid Interface Sci., (1999). 219, $225-240$. 
6. Brenan, K.E., Campbell, S.L., Petzold, L.R., Numerical Solution of Initial-Value Problems in Differential Algebraic Equations, (1996). SIAM, Philadelphia,

7. Bron, A.J., Diagnosis of dry eye, Surv. Ophthalmol., (2001). 45(S2), S221-S226.

8. Bron, A.J., Tiffany, J.M., Pseudoplastic materials as tear substitutes: an exercise in design, The Lacrimal System, van Bijsterveld, O.P, Lemp, M.A. \& Spinelli, D. (eds.), (1991). Amsterdam: Kugler \& Ghedini,

9. Burelbach, J.P., Bankoff, S.G., Davis, S.H., Nonlinear stability of evaporating/condensing liquid films, J. Fluid Mech., (1988). 195, 463494.

10. Creech, J.L., Do, L.T., Fatt, I., Radke, C.J., In vivo tear-film thickness determination and implications for tear-film stability, Curr. Eye Res., (1998). 17, 1058-1066.

11. Doughty, M.J., Laiquzzaman, M., Button, N.F., Video-assessment of tear meniscus height in elderly Caucasians and its relationship to the exposed ocular surface, Curr. Eye Res., (2001). 22, 420-426.

12. Ehlers, N., The precorneal film: Biomicroscopical, histological and chemical investigations, Acta Ophthalmol. Suppl., (1965). 81, 3-135.

13. Fatt, I., Weissman, B.A., Physiology of the Eye - An Introduction to the Vegetative Functions 2nd Edn., (1992). Butterworth-Heinemann, Stoneham, Boston,

14. Franck, C., Fatty layer of the precorneal film in the 'office eye syndrome', Acta Ophthalmol., (1991). 69, 737-743.

15. Holly, F.J., Formation and rupture of the tear film, Exp. Eye Res., (1973). 15, $515-525$.

16. Holly, F.J., Lemp, M.A., Tear physiology and dry eyes, Surv. Ophthalmol., (1977). 22, 69-87.

17. Joo, S.W., Davis, S.H., Bankoff, S.G., Long-wave instabilities of heated falling films: two-dimensional theory of uniform layers, J. Fluid Mech., (1991). 230, 117-146.

18. Lemp, M.A., Report of the National Eye Institute/industry workshop on clinical trials in dry eyes, CLAO J., (1995). 21, 221-232.

19. Mathers, W.D., Ocular evaporation in meibomian gland dysfunction and dry eye, Ophthalmology, (1993). 100, 347-351. 
20. McCulley, J.P., Shine, W., A compositional based model for the tear film lipid layer, Trans. Am. Ophth. Soc., (1997). 95, 79-93.

21. Miller, D., Measurement of the surface tension of tears, Arch. Ophthalmol., (1969). 82, 368-371.

22. Mishima, S., Some physiological aspects of the precorneal tear film, Arch. Ophthalmol., (1965). 73, 233-241.

23. Mishima, S., Maurice, D.M., The oily layer of the tear film and evaporation from the corneal surface, Exp. Eye Res., (1961). 1, 39-45.

24. Nagyová, B., Tiffany, J.M., Components responsible for the surface tension of human tears, Curr. Eye Res., (1999). 19, 4-11.

25. Naire, S., Braun, R.J., Snow, S.A., An insoluble surfactant model for a vertical draining free film with variable surface viscosity, Phys. Fluids, (2001). 13, 2492-2502.

26. Norn, M.S., Semiquantitative interference study of fatty layer of precorneal film, Acta Ophthalmol., (1979). 57, 766-774.

27. Panzarella, C.H., Davis, S.H., Bankoff S.G., Nonlinear dynamics in horizontal film boiling, J. Fluid. Mech., (2000). 402, 163-194.

28. Port, M.J.A., Asaria, T.S., The assessment of human tear volume, J. Br. Contact Lens Assoc., (1990). 13, 76-82.

29. Rolando, M., Refojo, M.F., Tear evaporimeter for measuring water evaporation rate from the tear film under controlled conditions in humans, Exp. Eye Res., (1983). 36, 25-33.

30. Rolando, M., Zierhut, M., The ocular surface and tear film and their dysfunction in dry eye disease, Surv. Ophthalmol., (2001). 45(S2), S203S210.

31. Schwartz, L.W., Roy, R.V., Modeling draining flow in mobile and immobile soap films, J. Colloid Interface Sci., (1999). 218, 309-323.

32. Sharma, A., Energetics of corneal epithelial cell-ocular mucus-tear film interactions: Some surface-chemical pathways of corneal defense, Biophys. Chem., (1993). 47, 87-99.

33. Sharma, A., Acid-base interactions in the cornea-tear film system: surface chemistry of corneal wetting, cleaning, lubrication, hydration and defense, J. Dispersion Sci. Technol., (1998). 19, 1031-1068. 
34. Sharma, A., Khanna, R., Reiter, G., A thin film analog of the corneal mucus layer of the tear film: an enigmatic long range non-classical DLVO interaction in the breakup of thin polymer films, Coll. Surf. B, (1999). 14, 223-235.

35. Sharma, A., Ruckenstein, E., An analytical nonlinear theory of thin film rupture and its application to wetting films, J. Colloid Interface Sci., (1986). 113, 456-479.

36. Sharma, A., Tiwari, S., Khanna, R., Tiffany, J.M., Hydrodynamics of meniscus-induced thinning of the tear film, Lacrimal Gland, Tear Film, and Dry Eye Syndromes 2, Sullivan, D.A., Dartt, D.A. \& Meneray, M.A. (eds.), (1998). New York: Plenum Press,

37. Tiffany, J.M., The viscosity of human tears, Int. Opthalmol., (1991). 15, $371-376$.

38. Wilson, E., Anatomy of Eye and Orbit (4th Edn.), (1954). Blakiston Co., New York,

39. Wilson, S.D.R., The drag-out problem in film coating theory, J. Eng. Math., (1982). 16, 209-221.

40. Wong, H., Fatt, I., Radke, C.J., Deposition and thinning of the human tear film, J. Colloid Interface Sci., (1996). 184, 44-51.

41. Yokoi, N., Bron, A.J., Tiffany, J.M., Kinoshita, S., Reflective meniscometry - A new field of dry eye assessment, Cornea, (2000). 19(S1), S37-S43. 


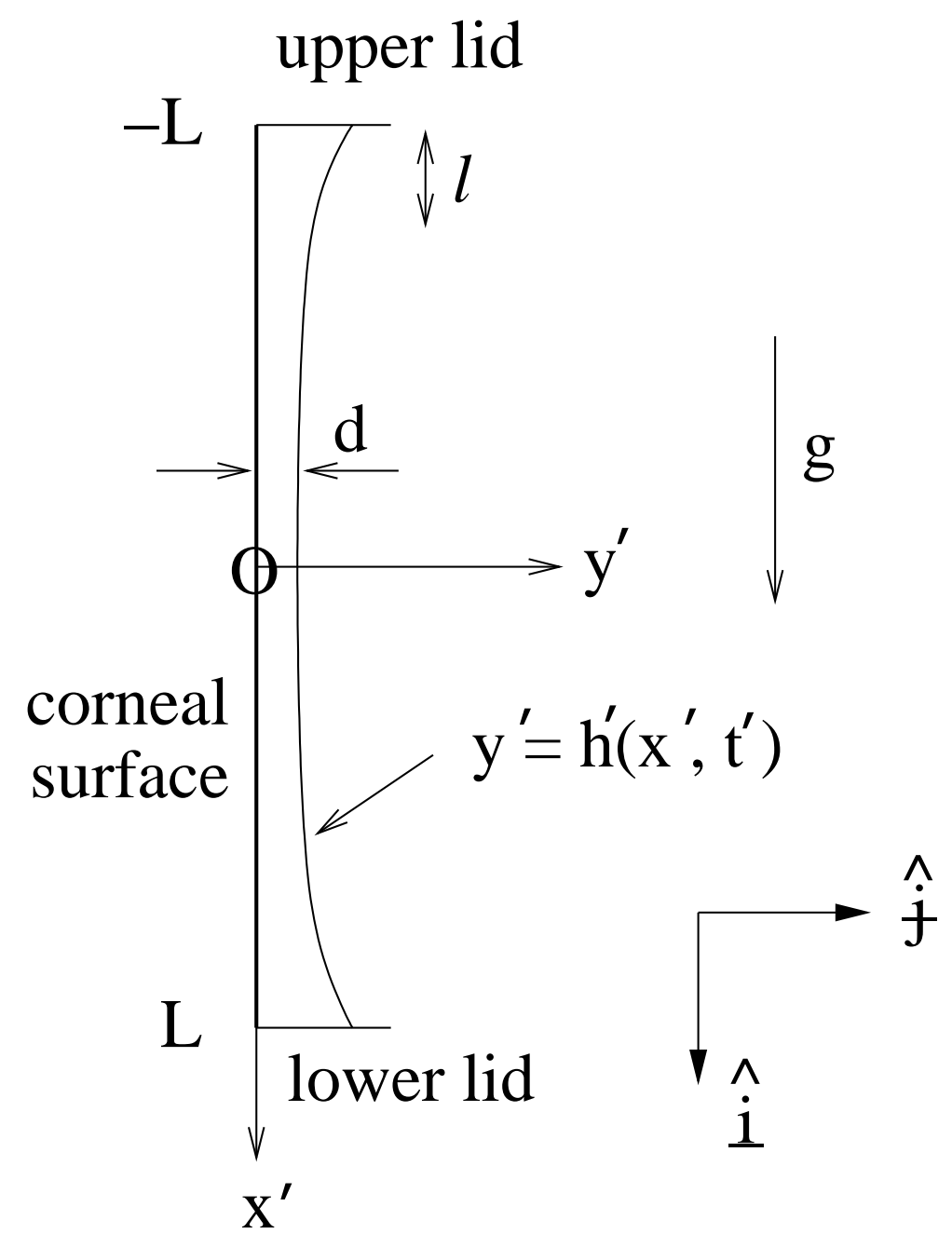

Figure 1: Schematic diagram of coordinate system used for tear film drainage model. 


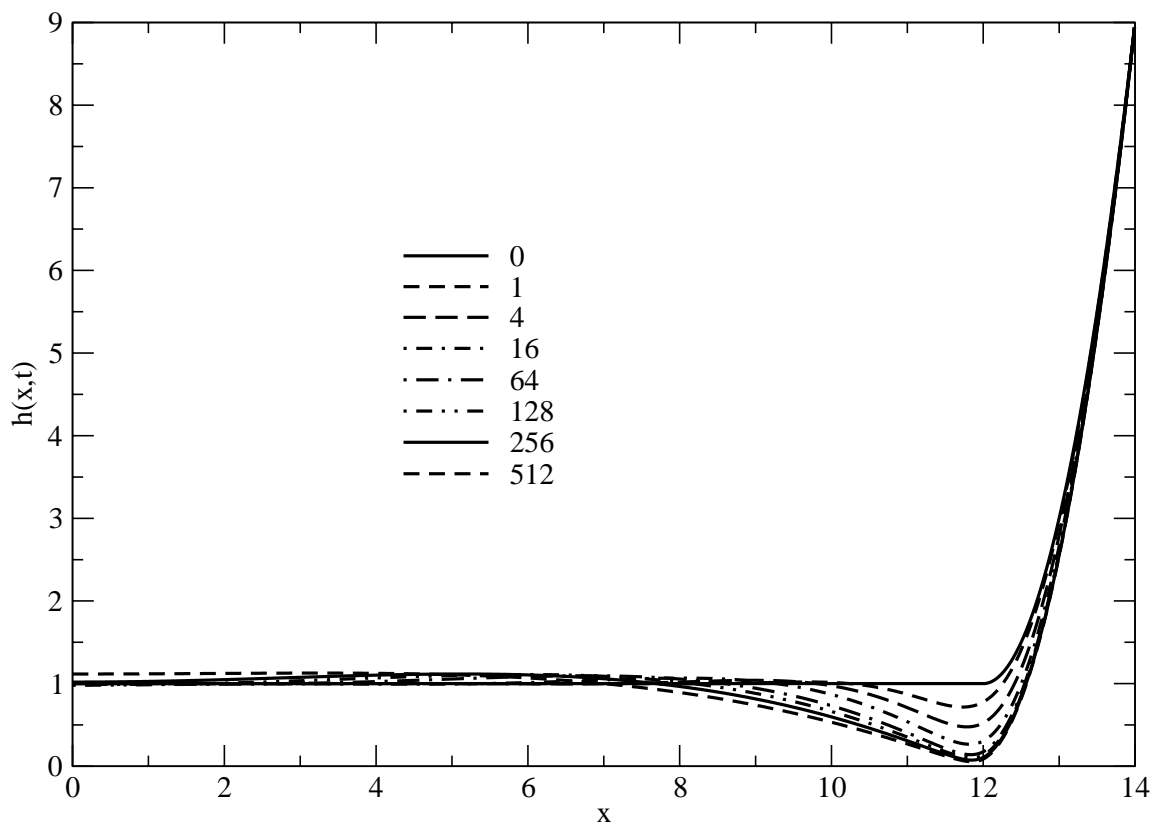

Figure 2: Numerical solution of (42) for the whole film $(-L \leq x \leq L)$ with no gravity or evaporation $\left(G=E=J_{0}=0\right)$ and $L=14$; only half of the film is shown because of the symmetry about $x=0$. The initial conditions have $\Delta h_{m}=\Delta x_{m}=2$. The boundary conditions are $h( \pm L, t)=9, h_{x x}( \pm L, t)=4$. 


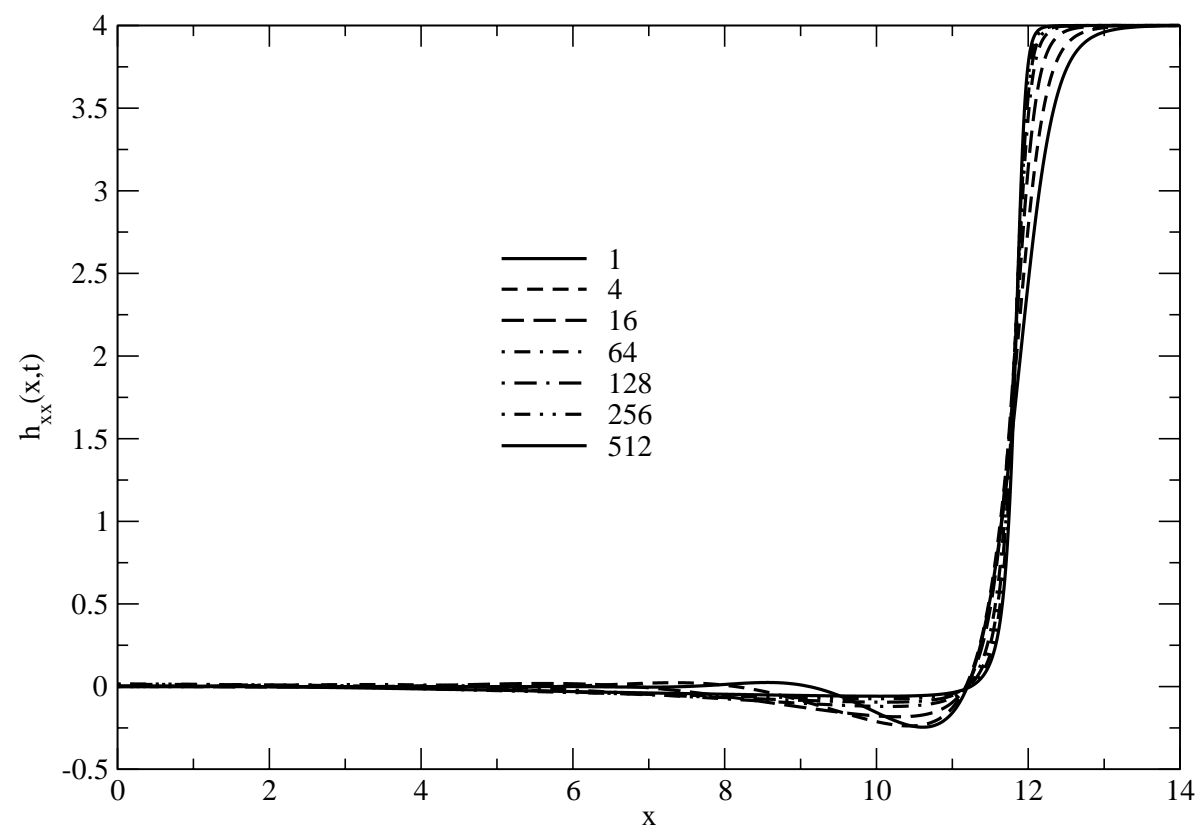

Figure 3: Curvature evolution with $G=E=J_{0}=0$ for the same data used for the results of Figure 2. The curvature appears to evolve towards a step change with time when the initial curvature in the meniscus is relatively large. 


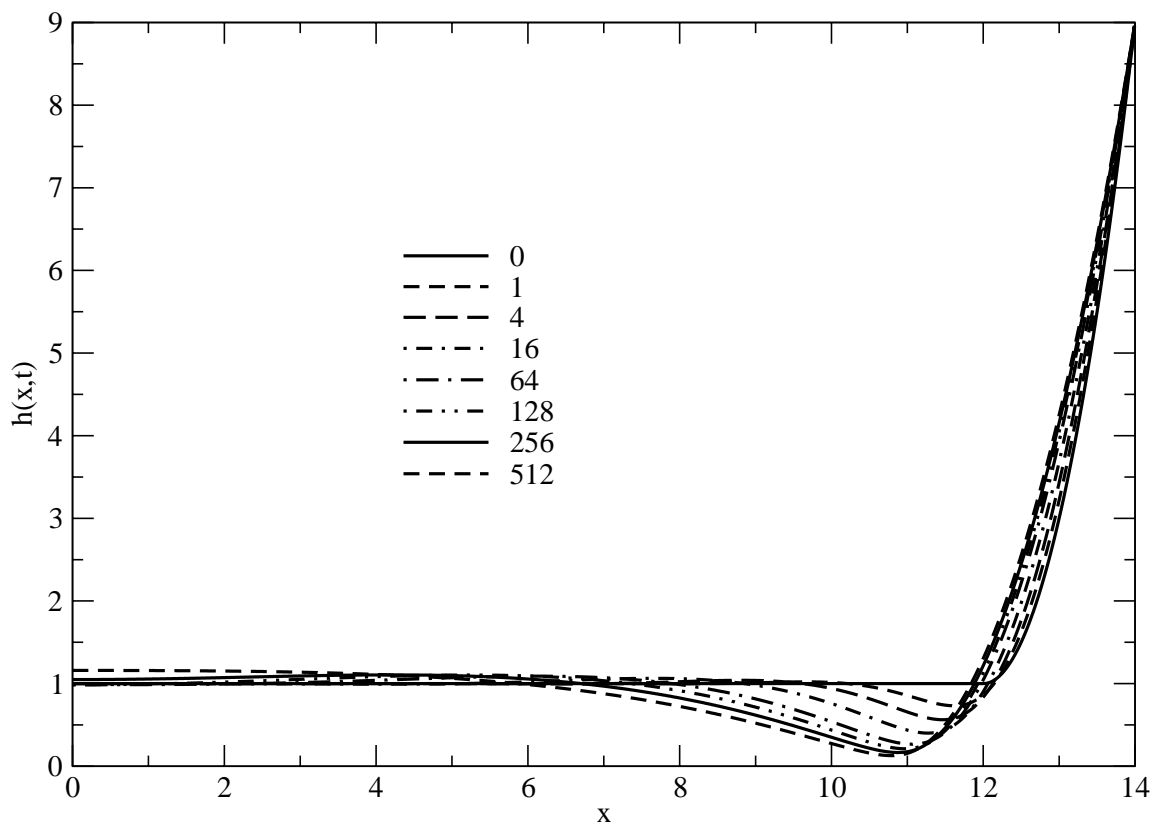

Figure 4: Computations with $G=E=J_{0}=0$ but with no flux boundary conditions. The minimum film thickness remains greater and evolves more slowly than in figure 2 , but this difference diminishes as the initial curvature of the meniscus in increased. 


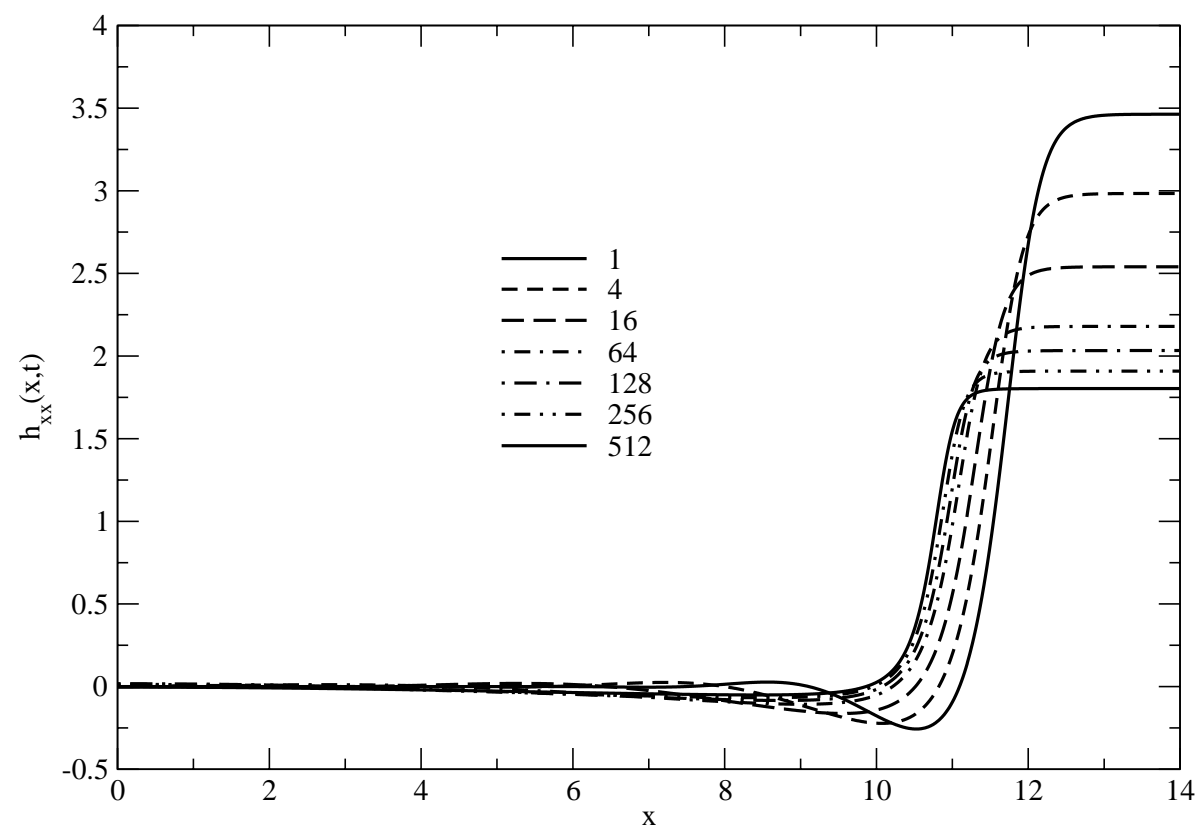

Figure 5: Curvature evolution with $G=E=J_{0}=0$, for the same data used in figure 4 . The curvature now decreases dramatically at the boundary compared to the results of figure 3 . 


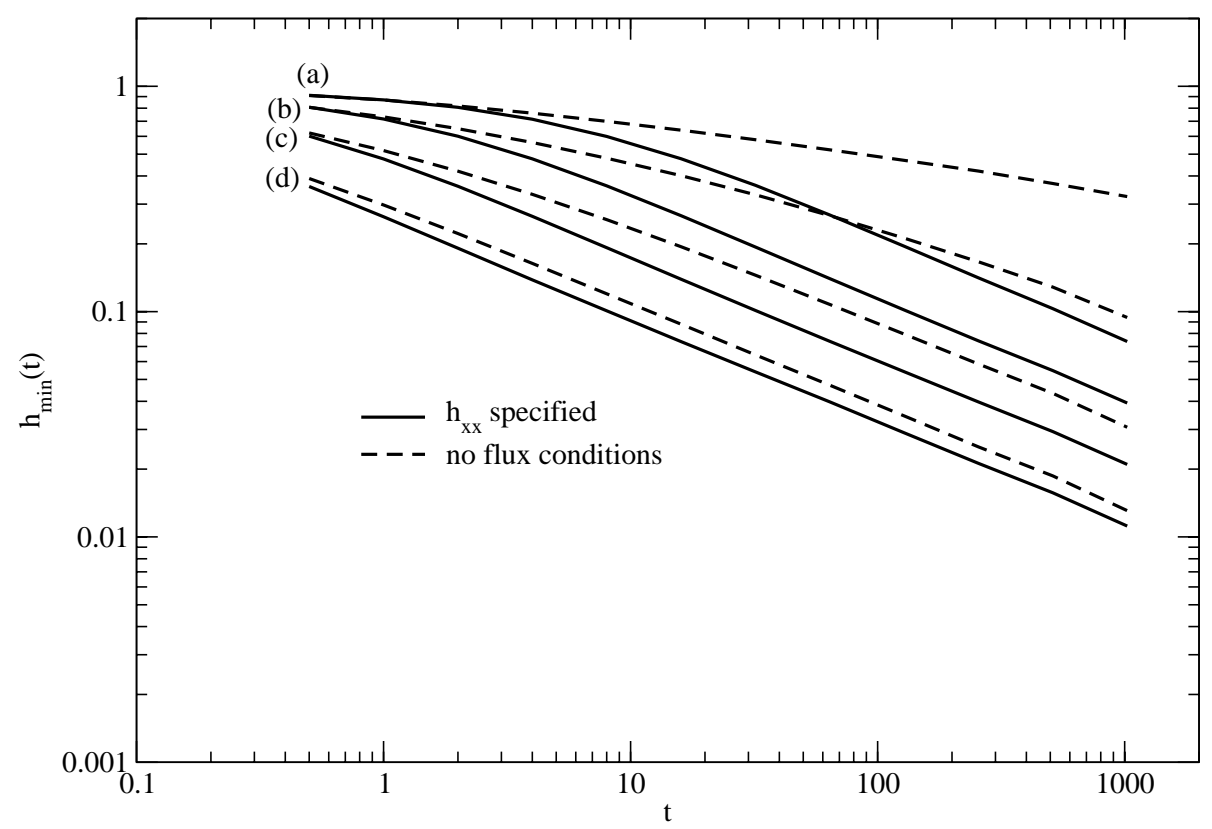

Figure 6: Minimum film thickness $h_{\min }(t)$ with $G=E=J_{0}=0$ for no flux and fixed curvature boundary conditions. The initial conditions are given by $\Delta x_{m}=2$ and: (a) $\Delta h_{m}=1$; (b) $\Delta h_{m}=2$; (c) $\Delta h_{m}=4$; (d) $\Delta h_{m}=8$. 




Figure 7: The case with no gravity $(G=0)$, but with evaporation $\left(J_{0}^{-1}=4930\right.$, $E=14.1) ; L=14, \Delta h_{m}=\Delta x_{m}=2$, and $h_{x x}=( \pm L, t)=4$. For clarity, only the interval $0<h<3$ is displayed. Note that evaporation causes the entire film thickness to decrease during the evolution. 


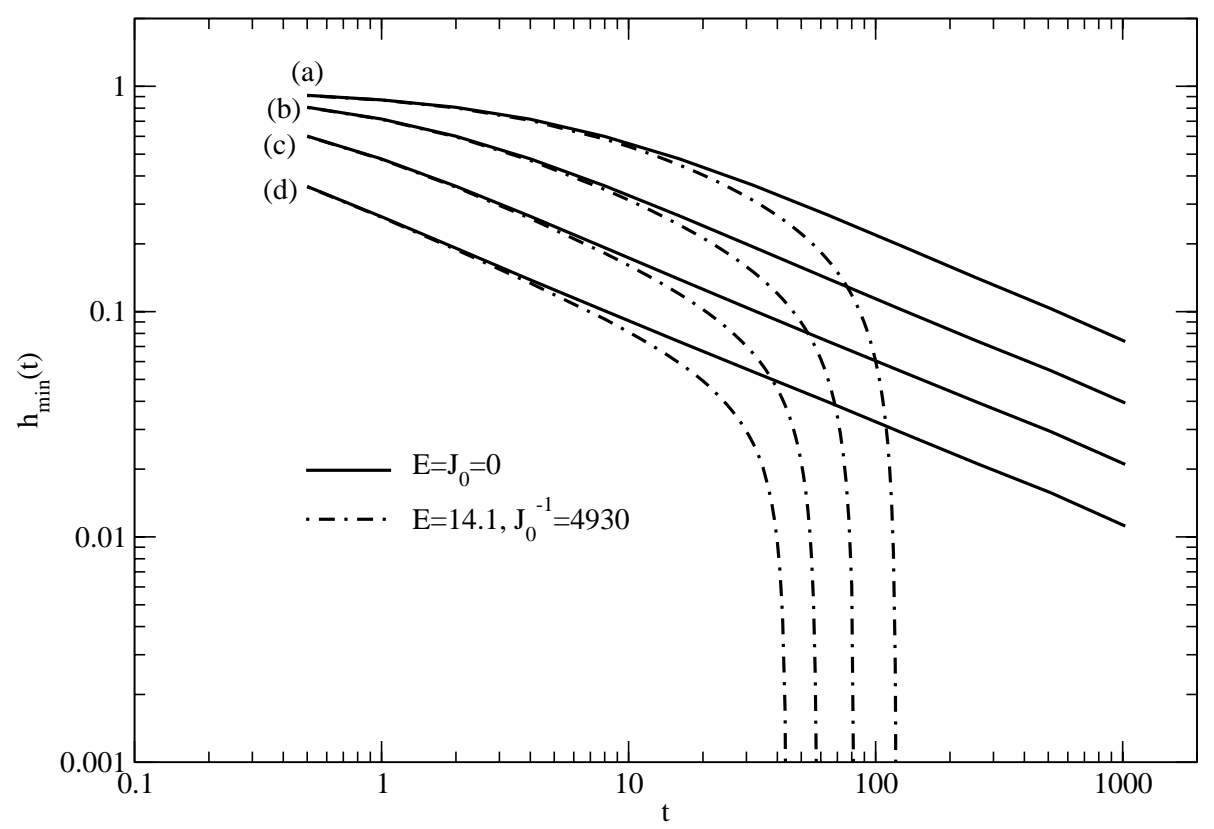

Figure 8: Minimum film thickness $h_{\min }(t)$ with $G=0$, without evaporation (solid line) and with evaporation (broken line) for fixed curvature boundary conditions with $\Delta x_{m}=2$ and: (a) $\Delta h_{m}=1$, (b) $\Delta h_{m}=2$, (c) $\Delta h_{m}=4$ (d) $\Delta h_{m}=8$. 


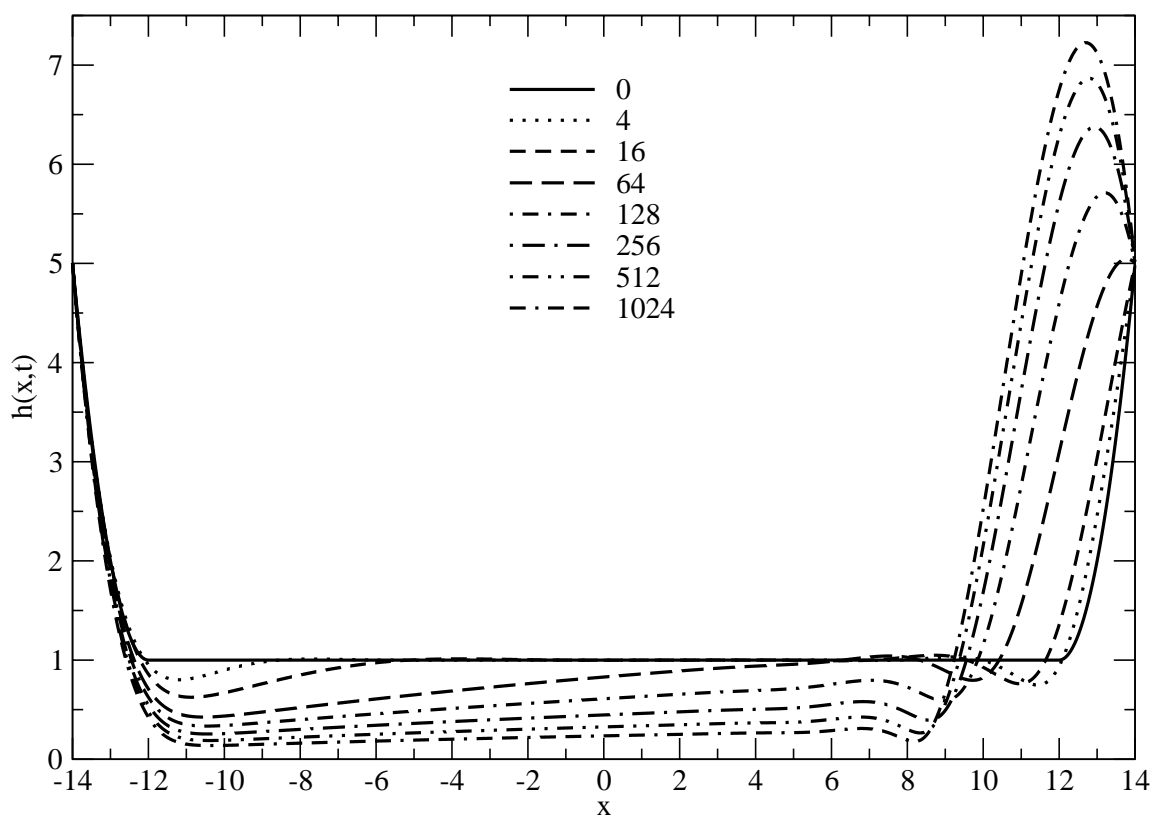

Figure 9: A case with gravity $(G=1)$ but no evaporation $\left(E=J_{0}=0\right)$, no flux boundary conditions and $\Delta h_{m}=1, \Delta x_{m}=2$. The film develops asymmetric menisci at the top and the bottom; gravity acts from left to right. The long middle region has very little curvature and is driven by gravity. The very small menisci prescribed initially allow for a bulge (the "tear pool") at the bottom of the film, which, at each time shown, is a good approximation to a static meniscus. 


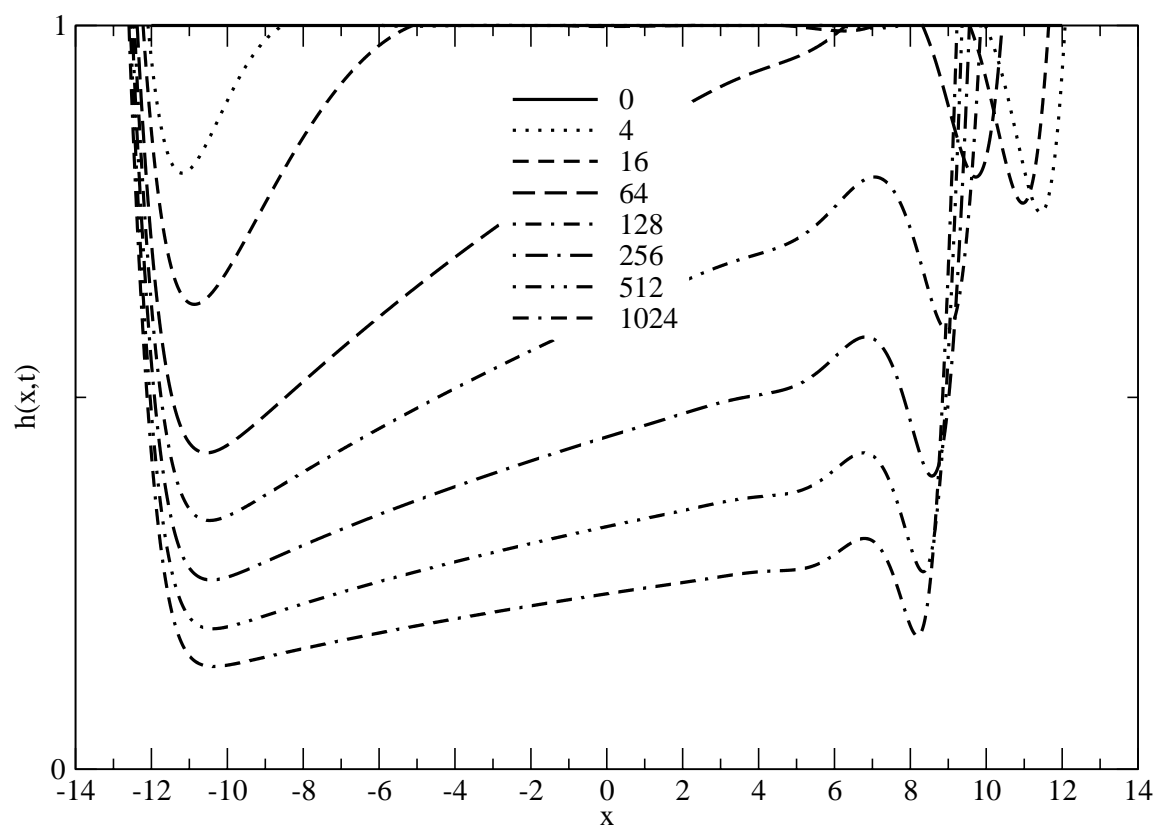

Figure 10: Zooming in on the results of figure $9\left(G=1, E=J_{0}=0\right.$, no flux boundary conditions, $\Delta h_{m}=1, \Delta x_{m}=2$ ). The thinnest region occurs near the top of the film; some oscillation is visible where the film drains into the lower meniscus. 


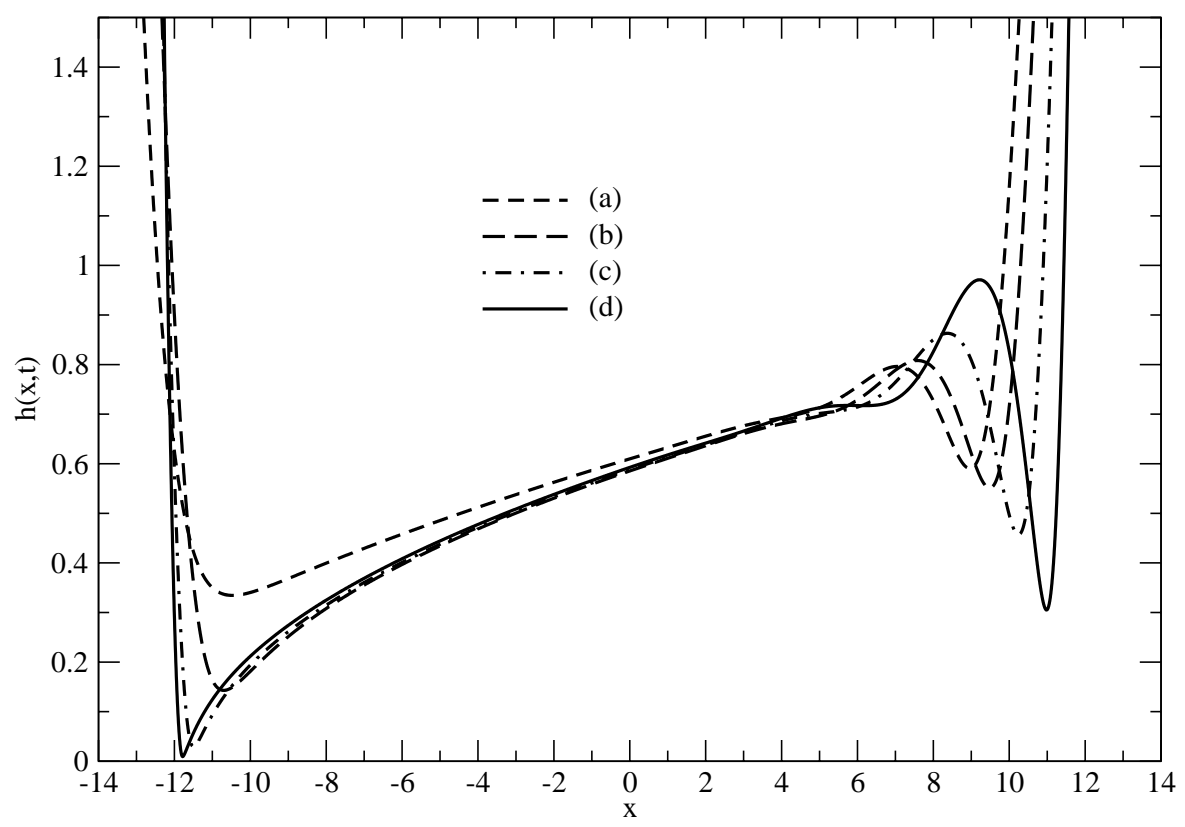

Figure 11: Results for four different initial conditions at $t=128(62 \mathrm{~s})$ are shown for $G=1, E=J_{0}=0$ : (a) $\Delta h_{m}=1$; (b) $\Delta h_{m}=2$; (c) $\Delta h_{m}=4$; (d) $\Delta h_{m}=8$. In all four cases we use $L=14, \Delta x_{m}=2$ and no flux boundary conditions are assumed. As the curvature of the initial menisci at the ends increases, the depletion of the top region is enhanced. 




Figure 12: Minimum film thickness $h_{\min }(t)$ with $G=1, E=J_{0}=0$ for no flux boundary conditions. The initial conditions are given by $\Delta x_{m}=2$ and: (a) $\Delta h_{m}=1$; (b) $\Delta h_{m}=2$; (c) $\Delta h_{m}=4$; (d) $\Delta h_{m}=8$. The minimum changes much more rapidly near to the top of the film. 


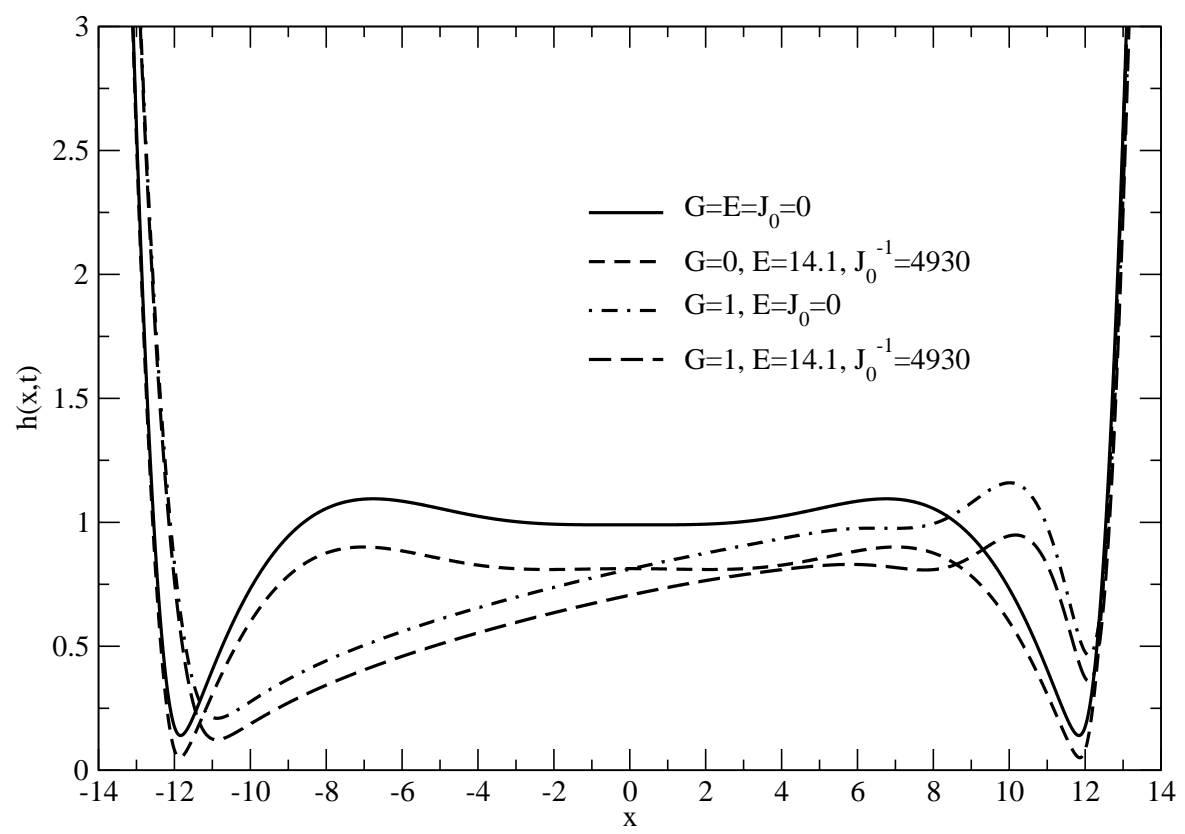

Figure 13: Comparison of different cases with and without evaporation and gravity for $\Delta h_{m}=\Delta x_{m}=2, L=14$ for $t=64$ (31s) and fixed curvature boundary conditions. The role of evaporation is more important as the film thins; the rate of thinning decreases in its absence. 


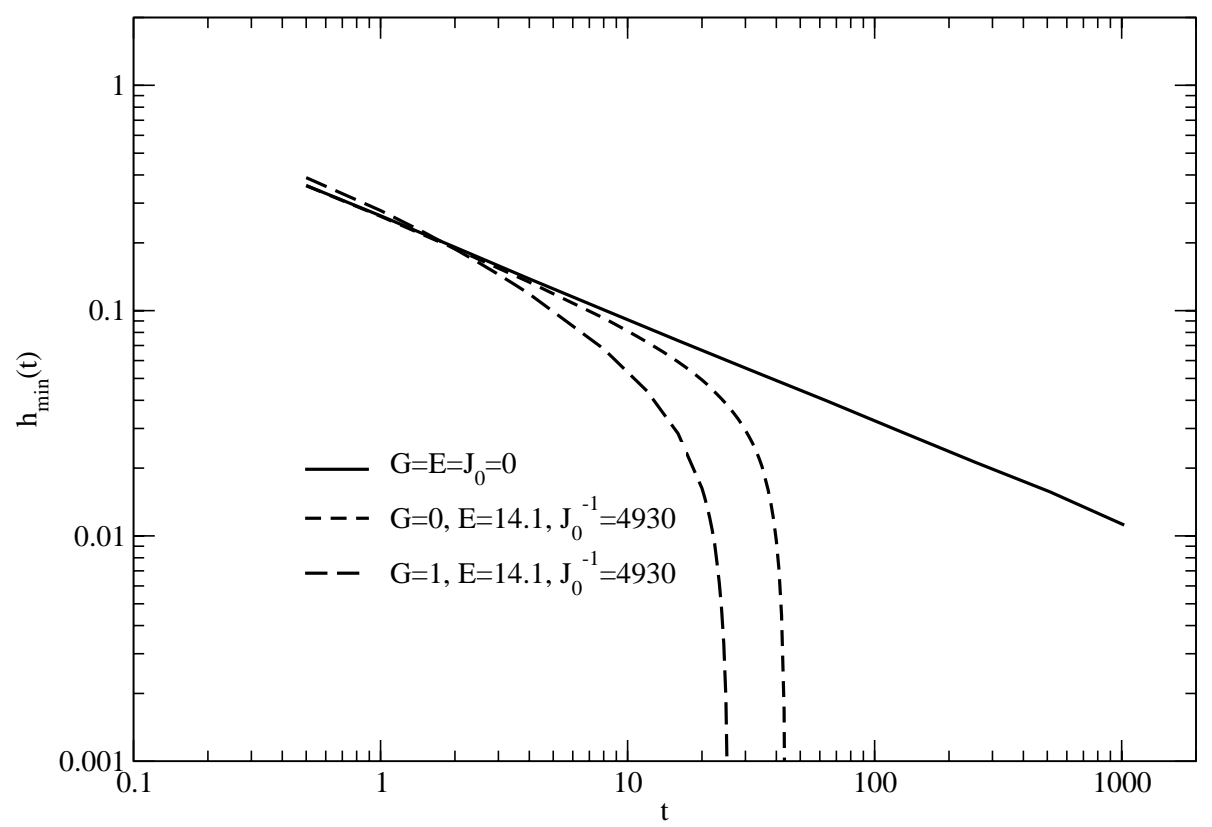

Figure 14: Comparison of cases with and without evaporation and gravity for $\Delta h_{m}=8, \Delta x_{m}=2, L=14$. Gravity and evaporation cooperate to significantly reduce the predicted BUT. 